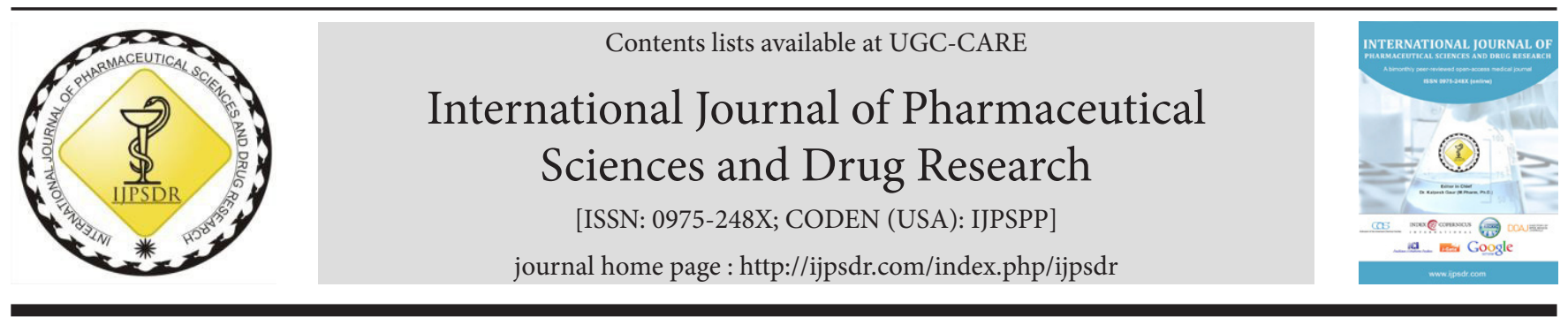

Research Article

\title{
Selection the Drug Efficacy of Oroidin Derivatives as Hsp90 Inhibitors by Computer Aided Drug Design Method
}

\author{
Subhajit Sarkar, Rajesh Kumar Das* \\ Department of Chemistry, University of North Bengal, Darjeeling, West Bengal, India.
}

\begin{tabular}{l} 
A R T I C L E I N F O \\
\hline Article history: \\
Received: 16 August, 2020 \\
Revised: 21 October, 2020 \\
Accepted: 29 October, 2020 \\
Published: 30 November, 2020 \\
Keywords: \\
ADME prediction, \\
DFT, \\
Hsp90, \\
Inhibitor, \\
Molecular docking, \\
Molecular descriptor properties. \\
Oroidin. \\
D0I: \\
10.25004/IJPSDR.2020.120607
\end{tabular}

\section{INTRODUCTION}

Cancer is a collection of related diseases that initiate the uncontrolled growth of abnormal cells with the ability to spread to adjoining or other parts of the body. According to a world health organization (WHO) report, it was estimated that cancer, 2nd leading cause of death globally, accounting for over 9.6 million deaths in 2018. ${ }^{[1]}$ Nowadays, research on alternative or combined therapies has received wide interest as traditional chemotherapy has a severe adverse effect during and after treatment. ${ }^{[2]}$ Hsp90 (heat shock protein 90) involves a significant role in cancer and presently, Hsp90 inhibitors have become intense research attention. ${ }^{[3]} \mathrm{Hsp} 90$ is a molecular

\begin{abstract}
A B S T R A C T
Heat shock protein 90 (Hsp90) is a conserved molecular chaperone associated with the regulation of hundreds of client proteins that are key drivers, regulators and promoters of numerous refractory diseases, therapies. Marine organisms are a rich source of pharmacologically important compounds, especially oroidin. pocket of Hsp90 and suppresses the ATPase activity of the protein. Natural product oroidin was selected as potent inhibitor of Hsp90, and its drug candidature was accordingly improved by substituting various functional groups. Virtual screenings were done through in silico studies, carried out on 39 derivatives of oroidin. DFT study was performed with Gaussian16, UB3LYP/6-311G++ (d, p) basis set to investigate the quantum mechanical parameters such as HOMO-LUMO energies, dipole moments. Derived parameters like ionization potential, electron affinity, softness-hardness, chemical potential, and electrophilicity index were macromolecule Hsp90 and recorded the best conformation's binding energy values out of nine in each docked Absorption, distribution, metabolism and excretion (ADME) predictions, molecular descriptor properties, and theoretical toxicity tests were evaluated using preADMET, molinspiration, and OSIRIS property possessing higher binding energies compare to the precursor oroidin. Seven of them qualified all the rules of drug candidature and could be safe in using as effective drugs for cancer treatment. This study suggests that these compounds could be synthesized for in vitro test and may lead to novel anticancer therapeutics.
\end{abstract}

\footnotetext{
"Corresponding Author: Rajesh Kumar Das

Address: Assistant Professor, Department of Chemistry, University of North Bengal, Darjeeling, West Bengal, 734013, India

Email $\bowtie:$ rajeshnbu@gmail.com

Tel.: +919434459238

Relevant conflicts of interest/financial disclosures: The authors declare that the research was conducted in the absence of any commercial or financial relationships that could be construed as a potential conflict of interest.

Copyright (C) 2020 Subhajit Sarkar etal. This is an open access article distributed under the terms of the Creative Commons Attribution- NonCommercialShareAlike 4.0 International License which allows others to remix, tweak, and build upon the work non-commercially, as long as the author is credited and the new creations are licensed under the identical terms.
}

chaperone protein that stabilizes proteins against heat stress, assists other proteins' incorrect folding, promotes protein degradation, prevents protein aggregation, and regulates apoptosis through interaction with mediators of apoptotic pathways. ${ }^{[4-6]}$ Functional human genes encoding, cystolic Hsp90 $\alpha / \beta$ are two isoforms of Hsp90, largely non-polar on the inside and polar on the outside nine helices and eight antiparallel beta-pleated sheets. ${ }^{[7-9]}$ The protein (Hsp90) regulates gene expression, proliferation, cellular signal transduction, and transcription networks, but dysregulation causes cell homeostasis. misfolding and overexpression of client proteins, which are responsible factors for the development of refractory diseases 
including cancer, inflammation, neurodegeneration, and viral infection like hepatitis $C$ and Chikungunya. ${ }^{[10-12]}$ Heat shock protein and its co-chaperones, stabilizing numerous growth factors receptors and signaling molecules, including PI3K/AKT proteins, inhibit both apoptotic and necrotic pathways. ${ }^{[13,14]}$ ATP binding is the basis of the Hsp90 chaperone cycle. Inhibition of this protein by ATPase inhibitors weakens the client proteins and may induce apoptosis, the programmed cell death, which maintains the healthy survival death balance in metazoan cells. ${ }^{[15,16]}$ The ATPase-binding site is the principal binding region of drugs targeting Hsp90. ${ }^{[17]}$ Protein kinase A (PKA) phosphorylates a single residueThr90 of Hsp90 $\alpha$ and stimulates its secretion and thus tumor metastasis. ${ }^{[18]}$ On the other hand, the inhibition of serinethreonine acidophilic kinase phosphorylates two serine residues Ser226 and Ser255, resulting in stabilization of Hsp90 $\beta$-apoptosome interaction, may contribute to chemo-resistance in leukemias. ${ }^{[19]}$ Upregulation of $\mathrm{Hsp} 90 \beta$ protein leads to lung cancer with the highest mortality rate among carcinogens and autoimmune ovarian failure disease, causing infertility. ${ }^{[20,21]}$

It is reported that some natural products and their derivatives target Hsp90-ATP binding sites and exhibit antitumor activity. ${ }^{[22]}$ Marine sponges are an abundant source of natural products and their varieties of chemical compounds escalated the development of pharmaceuticals. ${ }^{[23,24]}$ Oroidin (ODN), a pyrrole2-aminoimidazole alkaloid (Fig. 1), derived from a marine sponge Agelas oroides, has significant biological activities, including antitumor, immunosuppressive and adrenoceptor-related activities. ${ }^{[25-29]}$ It displayed potency against breast, ovarian, colon, pancreas, liver carcinoma, and leukemia. ${ }^{[30-33]}$ An experimental study ${ }^{[12]}$ reported that some synthesized oroidin analogs, screened against replicon models of two ribonucleic acid (RNA) viruses, hepatitis C virus (genus Hepacivirus) and Chikungunya virus (genus Alphavirus), could inhibit bacterial deoxyribonucleic acid (DNA) gyrase. This bacterial protein's ATP-binding site shows high structural similarity to the ATP-binding site of heat-shock protein 90 (Hsp90). Based on these remarkable pharmacological properties we have chosen oroidin as a ligand to inhibit Hsp90.

Currently, drug design technique demands a suitable screening of the compounds to measure pharmacophore activity, using the theoretical and computational

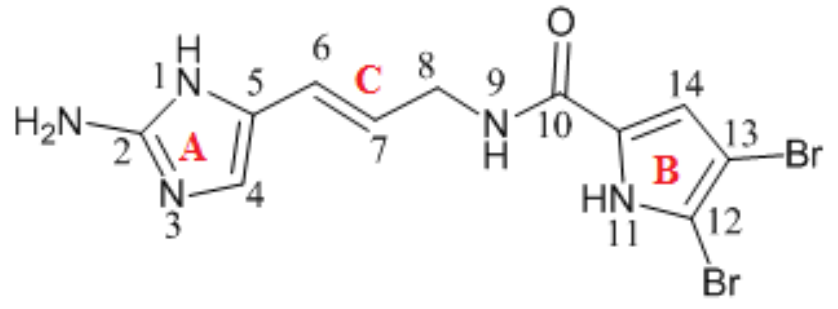

Fig 1: Structure of oroidin (ODN). approaches, before real-time experiments. Prediction of bioavailability, in vitro, and in vivo findings are enumerated by using the numerous physicochemical parameters. These properties are the basis of the idiosyncratic biological and physicochemical parameters of the compounds. Nevertheless, the complex structure of the whole drug compound faces difficulties in correlating with these parameters. ${ }^{[34]}$ The molecular docking method reveals the detailed possibilities of drug-receptor binding interactions. Thus the initial studies of receptor-ligand interaction have been gaining immense interest among logical drug designers. ${ }^{[35]}$

Based on these studies, we have modified oroidin to set up a library of 39 derivatives, undergoing molecular docking interaction with Hsp90 (PDB ID: 3NMQ), to improve the drug-like properties of the inhibitor ODN. In silico ADME predictions characterizing drug-likeness features were performed to screen the active biochemical derivatives. Our study's main goal was to find new chemical compounds, possessing better drug candidature than the natural oroidin, being potentially good inhibitors of Hsp90.

\section{METHODS AND MATERIALS}

\section{Preparation of Protein}

The 3-dimensional X-ray crystal structure of Hsp90 was collected from the Protein Data Bank (PDB ID: 3NMQ). Water and other chemical compounds were removed from the crystal structure before docking using Molegro Molecular viewer (MMV) program (CLC Bio, Qiagen Inc.). ${ }^{[36]}$

\section{Preparation of Ligand}

The software Chemdraw was used to draw the standard drug ODN and all its derivative ligands. ${ }^{[37]}$ The selected molecules obtained by various substitutions were shown in Table 1. All the explicit hydrogen was added at the time of preparation of ligands, including 2D-3D structure conversion. The compounds were optimized using DFT. ${ }^{[38,39]}$ Original structures and as well as the optimized structures of the four best derivatives of ODN are shown in Fig. 2, and the remaining are displayed in the supplementary Fig. S1.

\section{Density Function Theory (DFT)}

Gaussian16W ${ }^{[40]}$ suite of software was made to involve minimizing the compounds' energy content leading to geometrical optimization. Total energy, highest occupied molecular orbital (HOMO), lowest unoccupied molecular orbital (LUMO), corresponding energy-gap, and dipole moment were calculated (Table 2) using DFT model constrained to work under unrestricted Becke's three-parameter exchange potential and LeeYang-Parr correlation functional (UB3LYP) method with 6-311++G(d,p) basis set. ${ }^{[41,42]}$ 
Table 1: Binding energy of compounds having different

\begin{tabular}{|c|c|c|c|}
\hline Compounds & $\begin{array}{l}\text { Substituted } \\
\text { groups }\end{array}$ & Substituted by & $\begin{array}{l}\Delta G_{\text {Binding }} \\
\text { (kcal) } \\
\text { mol) }\end{array}$ \\
\hline ODN1 & $7-\mathrm{H}$ & 7- $\mathrm{NH}_{2}$ & -12.07 \\
\hline ODN2 & 6-H,7-H & 6- $\mathrm{NH}_{2}, 7-\mathrm{NH}_{2}$ & -11.94 \\
\hline ODN3 & $7-\mathrm{H}$ & 7- $\mathrm{CONH}_{2}$ & -12.63 \\
\hline ODN4 & 6-H,7-H & 6- $\mathrm{CONH}_{2}, 7-\mathrm{CONH}_{2}$ & -12.33 \\
\hline ODN5 & $7-\mathrm{H}$ & $7-\mathrm{OH}$ & -12.54 \\
\hline ODN6 & 6-H,7-H & 6-OH,7-OH & -10.97 \\
\hline ODN7 & 7-H & 7-COOH & -12.60 \\
\hline ODN8 & 6-H,7-H & 6-COOH,7-COOH & -11.04 \\
\hline ODN9 & 7-H & $7-\mathrm{F}$ & -11.26 \\
\hline ODN10 & 7-H & $7-\mathrm{CF}_{3}$ & -9.87 \\
\hline ODN11 & 7-H & $7-\mathrm{NO}_{2}$ & -11.37 \\
\hline ODN12 & $13-\mathrm{Br}$ & $13-\mathrm{PO}_{3} \mathrm{H}_{2}$ & -12.34 \\
\hline ODN13 & $8-\mathrm{H}, 13-\mathrm{Br}$ & 8- $\mathrm{PO}_{3} \mathrm{H}_{2}, 13-\mathrm{PO}_{3} \mathrm{H}_{2}$ & -11.60 \\
\hline ODN14 & $7-\mathrm{H}$ & $7-\mathrm{CN}$ & -10.42 \\
\hline ODN15 & $6-\mathrm{H}$ & $6-\mathrm{F}$ & -11.85 \\
\hline ODN16 & $6-\mathrm{H}$ & $6-\mathrm{CF}_{3}$ & -10.17 \\
\hline ODN17 & $13-\mathrm{Br}$ & $13-\mathrm{CONH}_{2}$ & -12.68 \\
\hline ODN18 & $2-\mathrm{NH}_{2}, 13-\mathrm{Br}$ & $2-\mathrm{CONH}_{2}, 13-\mathrm{CONH}_{2}$ & -13.18 \\
\hline ODN19 & $12-\mathrm{Br}$ & $12-\mathrm{OH}$ & -11.78 \\
\hline ODN20 & 12-Br,13-Br & $12-\mathrm{OH}, 13-\mathrm{OH}$ & -11.53 \\
\hline ODN21 & $13-\mathrm{Br}$ & $13-\mathrm{OCH}_{3}$ & -11.63 \\
\hline ODN22 & 7-H,13-Br & $7-\mathrm{OCH}_{3}, 13-\mathrm{OCH}_{3}$ & -11.72 \\
\hline ODN23 & $13-\mathrm{Br}$ & $13-\mathrm{COOH}$ & -12.25 \\
\hline ODN24 & $2-\mathrm{NH}_{2}, 13-\mathrm{Br}$ & 2-COOH,13-COOH & -12.23 \\
\hline ODN25 & $13-\mathrm{Br}$ & $13-\mathrm{F}$ & -11.50 \\
\hline ODN26 & $13-\mathrm{Br}$ & $13-\mathrm{NO}_{2}$ & -11.60 \\
\hline ODN27 & 13-Br,14-H & 13-H,14-Br & -11.56 \\
\hline ODN28 & 13-Br,14-H & $13-\mathrm{H}, 14-\mathrm{OH}$ & -11.28 \\
\hline ODN29 & 13-Br,14-H & $13-\mathrm{H}, 14-\mathrm{OCH}_{3}$ & -12.08 \\
\hline ODN30 & $2-\mathrm{NH}_{2}, 4-\mathrm{H}$ & $2-\mathrm{C}, 4-\mathrm{NH}_{2}$ & -12.39 \\
\hline ODN31 & $2-\mathrm{NH}_{2}, 4-\mathrm{H}, 7-\mathrm{H}$ & $2-\mathrm{C}, 4-\mathrm{NH}_{2}, 7-\mathrm{OH}$ & -11.83 \\
\hline ODN32 & $\begin{array}{l}2-\mathrm{NH}_{2}, 4-\mathrm{H}, 13- \\
\mathrm{Br}\end{array}$ & $2-\mathrm{H}, 4-\mathrm{NH}_{2}, 13-\mathrm{NH}_{2}$ & -11.66 \\
\hline ODN33 & $10-C=0$ & $10-\mathrm{C}(\mathrm{OH})$ & -10.88 \\
\hline ODN34 & $10-C=0$ & $10-\mathrm{C}(\mathrm{COOH})$ & -9.91 \\
\hline ODN35 & $10-C=0$ & $10-\mathrm{C}\left(\mathrm{OCH}_{3}\right)$ & -9.50 \\
\hline ODN36 & $10-C=0$ & $10-\mathrm{C}\left(\mathrm{CONH}_{2}\right)$ & -11.89 \\
\hline ODN37 & $6-\mathrm{H}$ & $6-\mathrm{CN}$ & -11.41 \\
\hline ODN38 & $10-C=0$ & $10-\mathrm{C}\left(\mathrm{CF}_{3}\right)$ & -11.88 \\
\hline ODN39 & 7-H & 7-CH(OH)COOH & -9.88 \\
\hline
\end{tabular}
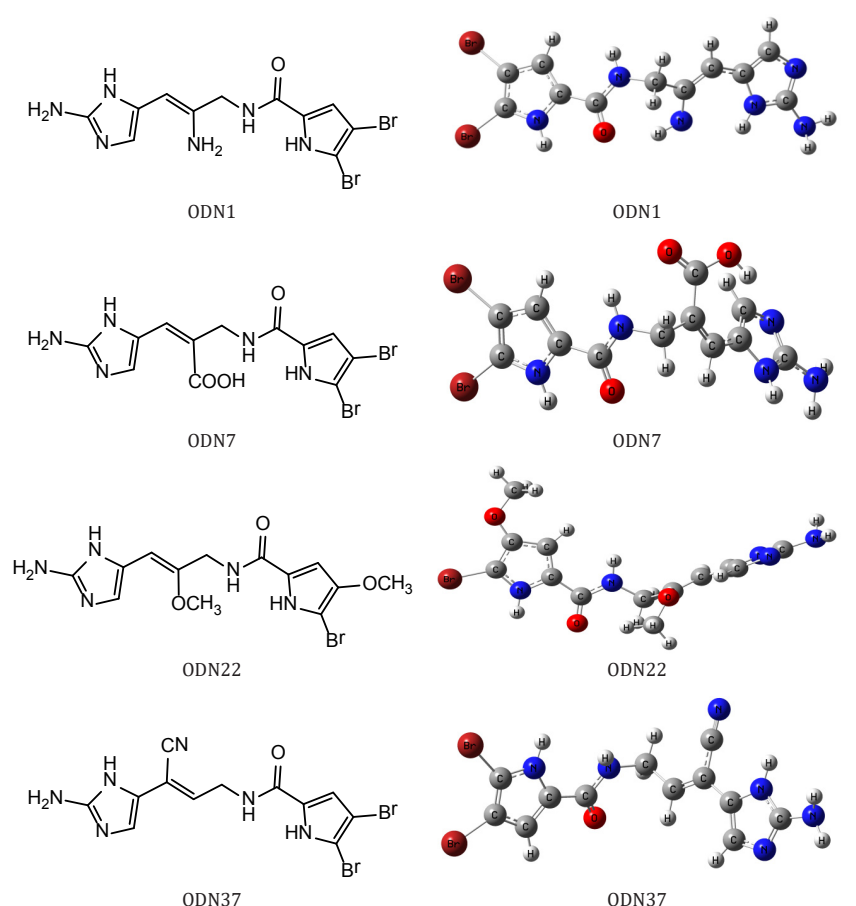

Fig 2: Compounds having, (a) normal structures and (b) optimized structures.

\section{Toxicity and ADME Parameters}

The overall toxicity of active derivative compounds was predicted by OSIRIS property explorer (http://www. organic-chemistry.org/prog/peo/).which indicates mutagenic, tumorigenic, irritant, and reproductive effect. Smiles were generated by SWISS ADME explorer.

Pre-ADMET (http://preadmet.bmdrc.org/) server was being exercised to explore drug-likeness properties and ADME profile (Absorption, distribution, metabolism, and excretion) of all the designed compounds. The extensive studies of $\log$ P, solubility, topological polar surface area (TPSA), molecular weight (MW), drug-likeness, drugscore, and the number of violations to Lipinski's rule had been carried out by Molinspiration (http://www. molinspiration.com)and OSIRIS program.

\section{Docking Method}

Auto dock tools 4.0 (ADT) ${ }^{[43]}$ interface program was used to predict the binding mode of ODN and its derivatives into the binding site of the receptor protein Hsp90 $\beta$ (PDB ID: $3 N M Q)$. The protein was made ready by checking, repairing missing atoms, adding all the missing hydrogen with no bond order, and using the ADT's graphical interface. To continue with the rigid docking process, the ligands were prepared as PDB files from all the optimized Gaussian output. Active torsions were kept to the maximum number of atoms. The grid box was so constructed that active sites of the receptor based on amino acid residues, were covered. The grid box size was maintained at 94, 94, $94 \AA$ ( $x, y$, and $z$ respectively) and center at $0.076,15.013,20.513$ 
Drug Efficacy of Oroidin Derivatives as Hsp90 Inhibitors

$$
\begin{aligned}
& \text { ODN2 } \\
& \text { 烈 }
\end{aligned}
$$

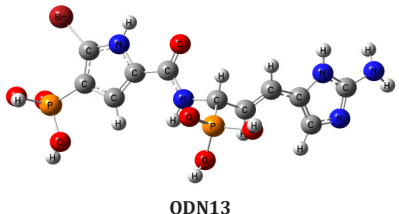

$$
\begin{aligned}
& \text { (1) } \\
& \text { (2) } \\
& \text { ODN3 } \\
& \text { ODN4 } \\
& \text { ODN4 } \\
& \text { (n) }
\end{aligned}
$$

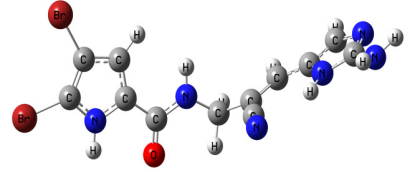

$$
\begin{aligned}
& \text { (1) }
\end{aligned}
$$

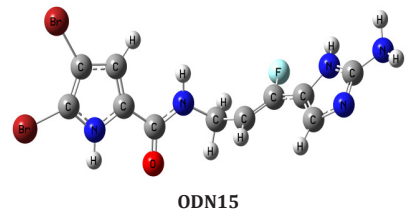

$$
\begin{aligned}
& \text { (1) } \\
& \text { (3) } \\
& \text { (1) }
\end{aligned}
$$

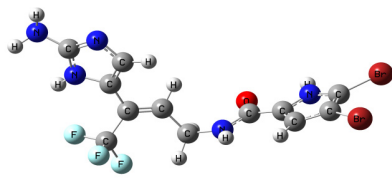

$$
\begin{aligned}
& \text { (1) }
\end{aligned}
$$

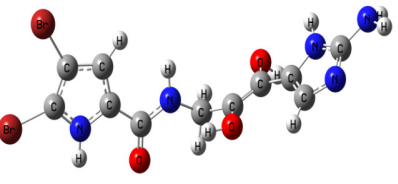

$$
\begin{aligned}
& \text { (c) } \\
& \text { 政 } \\
& \text { (1) } \\
& \text { (3) } \\
& \text { ODN9 } \\
& \text { (1) } \\
& \text { ODN17 } \\
& \text { ODN10 }
\end{aligned}
$$

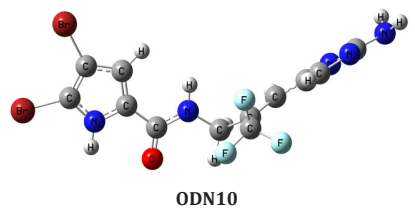

$$
\begin{aligned}
& \text { ODN19 }
\end{aligned}
$$

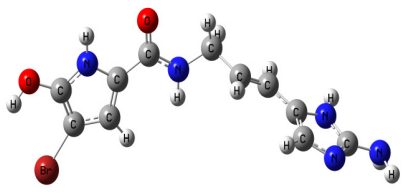

$$
\begin{aligned}
& \text { (1) } \\
& \text { - } 3 \text { (2) }
\end{aligned}
$$
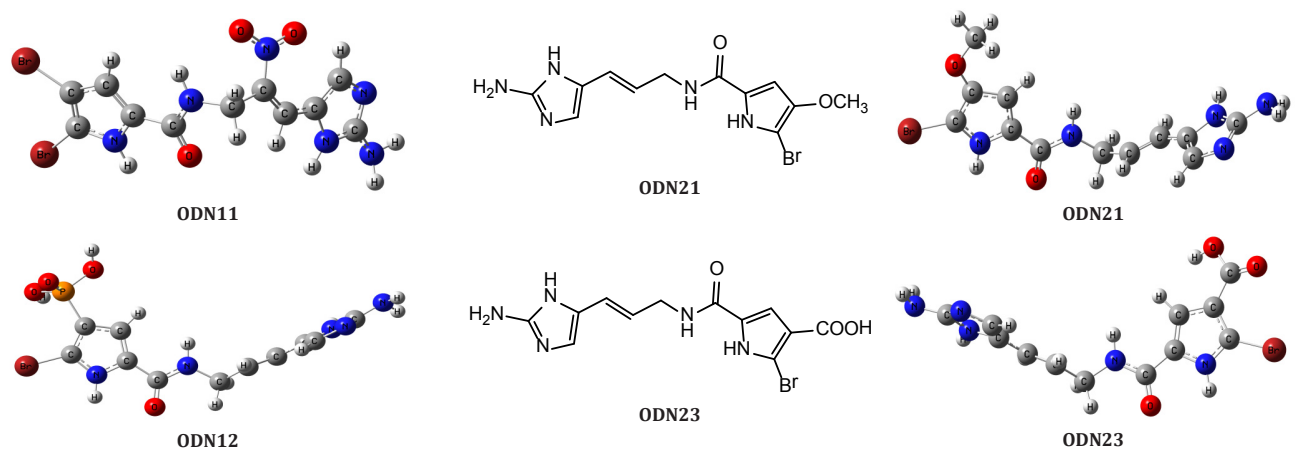

Fig S1: Compounds having, (a) normal structure and (b) optimized structure. 


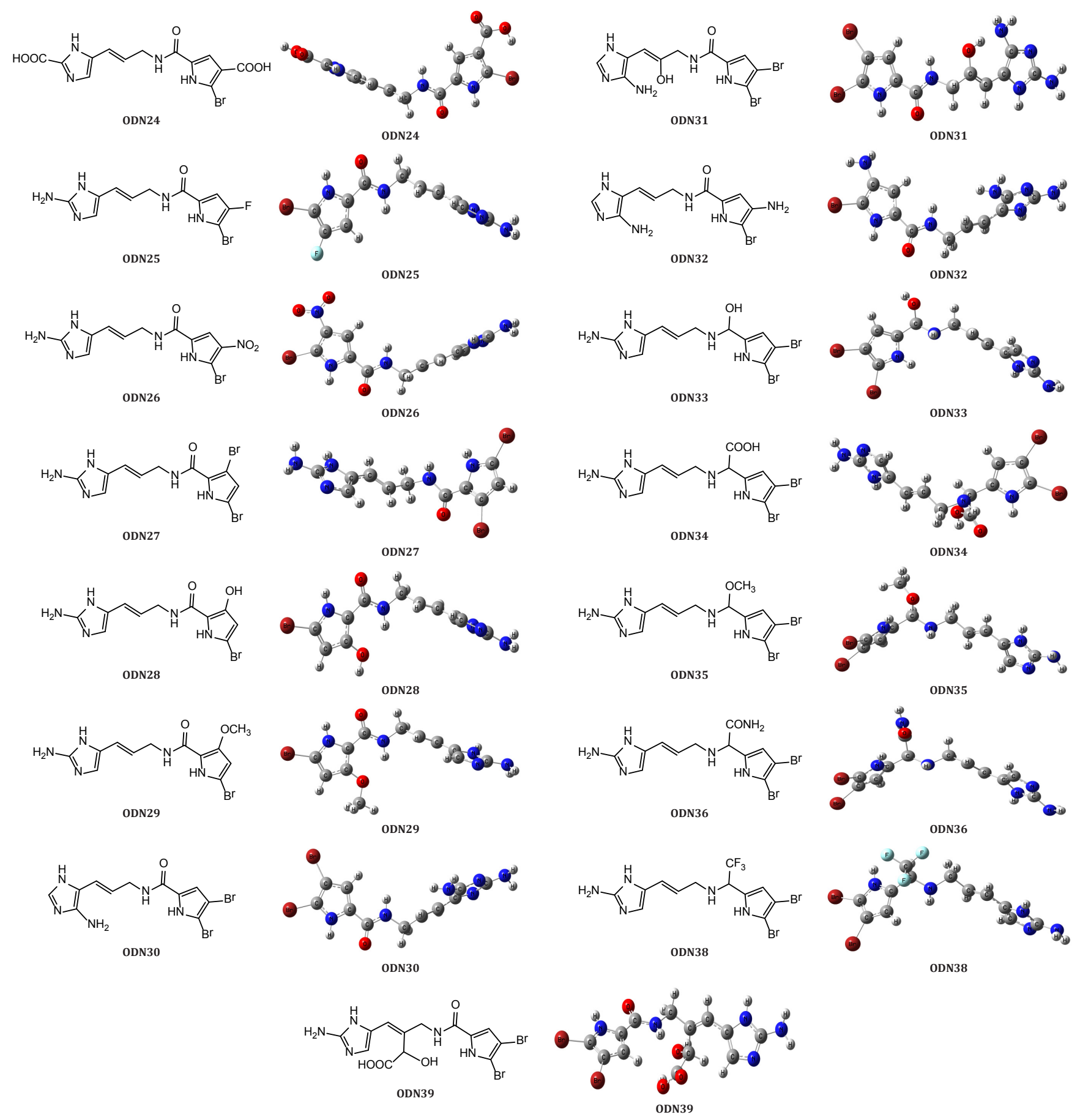

Fig S1: Compounds having, (a) normal structure and (b) optimized structure.

$(\mathrm{x}, \mathrm{y}$, and $\mathrm{z}$ respectively) with grid point spacing 0.525 $\AA$ A. Thirty-nine separate molecular docking experiments were performed, keeping the Lamarckian genetic algorithm (LGA) and other parameters in default mode. Nine conformations were generated for each ligand and the docked compounds with maximum cluster size as well as maximum binding energy were considered for advanced studies. The docking output results were transformed into .pdb format from .dlg, using python script (PMV 1.5.6). ${ }^{[44]}$

\section{RESULTS}

The results (Table 1) executed by molecular docking revealed that the docked complex of twenty-eight derived compounds has higher binding energy (docked with PDB: 3NMQ) than ODN (-11.58 Kcal/mol). HOMO-LUMO energies and dipole moments of all forty molecules were listed in Table (2) from DFT analysis. The derived physical parameters like ionization potential (I), electron affinity (A), global hardness $(\eta)$, softness 
Drug Efficacy of Oroidin Derivatives as Hsp90 Inhibitors

Table 2: Calculated energy values of oroidin and different derived compounds using UB3LYP/6-311++G(d,p).

\begin{tabular}{|c|c|c|c|c|c|}
\hline Compounds & $E_{\text {Номо }}(\mathrm{eV})$ & $E_{\text {LUMO }}(\mathrm{eV})$ & Band gap $\left(\Delta E_{H L}\right)(\mathrm{eV})$ & $E_{\text {TOTAL }} \times 10^{4}(\mathrm{eV})$ & Dipole moment (Debye) \\
\hline ODN & -5.67 & -1.38 & 4.29 & -16.12 & 2.24 \\
\hline ODN1 & -5.05 & -1.59 & 3.46 & -16.27 & 2.04 \\
\hline ODN2 & -5.26 & -1.23 & 4.03 & -16.42 & 3.81 \\
\hline ODN3 & -6.07 & -2.02 & 4.05 & -16.58 & 6.01 \\
\hline ODN4 & -5.85 & -1.86 & 3.99 & -17.04 & 7.40 \\
\hline ODN5 & -5.20 & -1.66 & 3.54 & -16.32 & 2.82 \\
\hline ODN6 & -5.27 & -1.53 & 3.74 & -16.53 & 4.19 \\
\hline ODN7 & -6.25 & -2.34 & 3.91 & -16.63 & 7.95 \\
\hline ODN8 & -6.32 & -2.68 & 3.64 & -17.14 & 7.06 \\
\hline ODN9 & -5.54 & -1.41 & 4.13 & -16.39 & 3.15 \\
\hline ODN10 & -6.03 & -1.92 & 4.11 & -17.04 & 7.52 \\
\hline ODN11 & -6.21 & -2.84 & 3.37 & -16.67 & 7.74 \\
\hline ODN12 & -5.67 & -1.45 & 4.22 & -10.66 & 8.48 \\
\hline ODN13 & -5.66 & -1.56 & 4.10 & -12.21 & 4.45 \\
\hline ODN14 & -6.03 & -2.19 & 3.84 & -16.37 & 3.62 \\
\hline ODN15 & -5.82 & -1.38 & 4.44 & -16.39 & 4.57 \\
\hline ODN16 & -5.90 & -1.66 & 4.24 & -17.04 & 2.92 \\
\hline ODN17 & -5.66 & -1.39 & 4.27 & -9.57 & 8.30 \\
\hline ODN18 & -6.47 & -1.88 & 4.59 & -9.88 & 3.82 \\
\hline ODN19 & -5.53 & -1.16 & 4.37 & -9.32 & 4.69 \\
\hline ODN20 & -5.48 & -1.08 & 4.40 & -2.52 & 5.12 \\
\hline ODN21 & -5.57 & -1.20 & 4.37 & -9.43 & 6.20 \\
\hline ODN22 & -5.21 & -1.19 & 4.02 & -9.74 & 5.07 \\
\hline ODN23 & -5.73 & -1.61 & 4.12 & -9.63 & 8.18 \\
\hline ODN24 & -6.61 & -2.15 & 4.46 & -9.99 & 4.08 \\
\hline ODN25 & -5.62 & -1.35 & 4.27 & -9.38 & 5.35 \\
\hline ODN26 & -5.72 & -2.65 & 3.07 & -9.67 & 6.87 \\
\hline ODN27 & -5.56 & -1.32 & 4.24 & -16.12 & 7.57 \\
\hline ODN28 & -5.45 & -1.00 & 4.45 & -9.32 & 5.07 \\
\hline ODN29 & 5.44 & -1.01 & 4.43 & -9.43 & 5.91 \\
\hline ODN30 & -5.10 & -1.21 & 3.89 & -16.27 & 5.41 \\
\hline ODN31 & -5.31 & -1.10 & 4.21 & -16.47 & 5.61 \\
\hline ODN32 & -5.00 & -0.96 & 4.04 & -9.42 & 5.65 \\
\hline ODN33 & -5.54 & -1.03 & 4.51 & -16.12 & 5.79 \\
\hline ODN34 & -5.54 & -1.12 & 4.42 & -16.43 & 6.36 \\
\hline ODN35 & -5.44 & -0.97 & 4.47 & -16.23 & 5.65 \\
\hline ODN36 & -5.47 & -1.04 & 4.43 & -16.38 & 6.75 \\
\hline ODN37 & -5.92 & -2.20 & 3.72 & -16.37 & 4.25 \\
\hline ODN38 & -5.57 & -1.17 & 4.40 & -16.83 & 3.09 \\
\hline ODN39 & -5.97 & -1.47 & 4.50 & -16.94 & 8.43 \\
\hline
\end{tabular}

$(S)$, chemical potential $(\mu)$, electronegativity $(\sigma)$, and In this study we have found that all the compounds obey electrophilicity $(\omega)$ had an important role to predict the Lipinski's rule of five, ${ }^{[45]}$ majority of them qualified activity of the thirty-nine derived compounds of oroidin CMC like rule (except ODN2, ODN13) and WDI like rule (Table 3).

(Table 4). As per Table 5, most of the compounds, with 
Subhajit Sarkar et al.

Table 3: Global reactivity descriptors of oroidin and derived compounds.

\begin{tabular}{|c|c|c|c|c|c|c|c|}
\hline Compounds & $I(\mathrm{eV})$ & $A(\mathrm{eV})$ & $\mu(\mathrm{eV})$ & $\eta(\mathrm{eV})$ & $S\left(e V^{-1}\right)$ & $\sigma(\mathrm{eV})$ & $\omega(\mathrm{eV})$ \\
\hline ODN & 5.67 & 1.38 & -3.53 & 2.15 & 0.47 & 3.53 & 2.90 \\
\hline ODN1 & 5.05 & 1.59 & -3.32 & 1.73 & 0.58 & 3.32 & 3.19 \\
\hline ODN2 & 5.26 & 1.23 & -3.25 & 2.02 & 0.50 & 3.25 & 2.61 \\
\hline ODN3 & 6.07 & 2.02 & -4.05 & 2.03 & 0.49 & 4.05 & 4.04 \\
\hline ODN4 & 5.85 & 1.86 & -3.86 & 2.00 & 0.50 & 3.86 & 3.72 \\
\hline ODN5 & 5.20 & 1.66 & -3.43 & 1.77 & 0.56 & 3.43 & 3.32 \\
\hline ODN6 & 5.27 & 1.53 & -3.40 & 1.87 & 0.53 & 3.40 & 3.09 \\
\hline ODN7 & 6.25 & 2.34 & -4.30 & 1.96 & 0.51 & 4.30 & 4.72 \\
\hline ODN8 & 6.32 & 2.68 & -4.50 & 1.82 & 0.55 & 4.50 & 5.56 \\
\hline ODN9 & 5.54 & 1.41 & -3.48 & 2.07 & 0.48 & 3.48 & 2.92 \\
\hline ODN10 & 6.03 & 1.92 & -3.98 & 2.06 & 0.49 & 3.98 & 3.84 \\
\hline ODN11 & 6.21 & 2.84 & -4.53 & 1.69 & 0.59 & 4.53 & 6.08 \\
\hline ODN12 & 5.67 & 1.45 & -3.56 & 2.11 & 0.47 & 3.56 & 3.00 \\
\hline ODN13 & 5.66 & 1.56 & -3.61 & 2.05 & 0.49 & 3.61 & 3.18 \\
\hline ODN14 & 6.03 & 2.19 & -4.11 & 1.92 & 0.52 & 4.11 & 4.40 \\
\hline ODN15 & 5.82 & 1.38 & -3.60 & 2.22 & 0.45 & 3.60 & 2.92 \\
\hline ODN16 & 5.90 & 1.66 & -3.78 & 2.12 & 0.47 & 3.78 & 3.37 \\
\hline ODN17 & 5.66 & 1.39 & -3.53 & 2.14 & 0.47 & 3.53 & 2.91 \\
\hline ODN18 & 6.47 & 1.88 & -4.18 & 2.30 & 0.44 & 4.18 & 3.80 \\
\hline ODN19 & 5.53 & 1.16 & -3.35 & 2.19 & 0.46 & 3.35 & 2.56 \\
\hline ODN20 & 5.48 & 1.08 & -3.28 & 2.20 & 0.45 & 3.28 & 2.45 \\
\hline ODN21 & 5.57 & 1.20 & -3.39 & 2.19 & 0.46 & 3.39 & 2.62 \\
\hline ODN22 & 5.21 & 1.19 & -3.20 & 2.01 & 0.50 & 3.20 & 2.55 \\
\hline ODN23 & 5.73 & 1.61 & -3.67 & 2.06 & 0.49 & 3.67 & 3.27 \\
\hline ODN24 & 6.61 & 2.15 & -4.38 & 2.23 & 0.45 & 4.38 & 4.30 \\
\hline ODN25 & 5.62 & 1.35 & -4.39 & 2.14 & 0.47 & 3.49 & 2.84 \\
\hline ODN26 & 5.72 & 2.65 & -4.19 & 1.54 & 0.65 & 4.19 & 5.70 \\
\hline ODN27 & 5.56 & 1.32 & -3.44 & 2.12 & 0.47 & 3.44 & 2.79 \\
\hline ODN28 & 5.45 & 1.00 & -3.23 & 2.23 & 0.45 & 3.23 & 2.34 \\
\hline ODN29 & 5.44 & 1.01 & -3.23 & 2.22 & 0.45 & 3.23 & 2.35 \\
\hline ODN30 & 5.10 & 1.21 & -3.16 & 1.95 & 0.51 & 3.16 & 2.56 \\
\hline ODN31 & 5.31 & 1.10 & -3.21 & 2.11 & 0.48 & 3.21 & 2.44 \\
\hline ODN32 & 5.00 & 0.96 & -2.98 & 2.02 & 0.50 & 2.98 & 2.20 \\
\hline ODN33 & 5.54 & 1.03 & -3.29 & 2.26 & 0.44 & 3.29 & 2.39 \\
\hline ODN34 & 5.54 & 1.12 & -3.33 & 2.21 & 0.45 & 3.33 & 2.51 \\
\hline ODN35 & 5.44 & 0.97 & -3.21 & 2.24 & 0.45 & 3.21 & 2.30 \\
\hline ODN36 & 5.47 & 1.58 & -3.26 & 2.22 & 0.45 & 3.26 & 2.39 \\
\hline ODN37 & 5.92 & 2.20 & -4.06 & 1.86 & 0.54 & 4.06 & 4.43 \\
\hline ODN38 & 5.57 & 1.17 & -3.37 & 2.20 & 0.45 & 3.37 & 2.58 \\
\hline ODN39 & 5.97 & 1.47 & -3.72 & 2.25 & 0.44 & 3.72 & 3.08 \\
\hline
\end{tabular}

a few exceptions were appeared to have healthy oral (Table 7) and the result predicted that twenty-two bioavailability. Except for a few, all the ligands indicated excellent permeability (Table 6). The drug score and compounds have better drug score value, in the range of 0.45-0.94 than that of ODN (0.45). Toxicity plays a key role drug-likeness values of the ligands were also evaluated in the selection of a promising drug. Numerous studies 
Drug Efficacy of Oroidin Derivatives as Hsp90 Inhibitors

Table 4: Drug-likeness properties of oroidin and derived compounds.

\begin{tabular}{|c|c|c|c|c|}
\hline Compounds & $\mathrm{CMC}^{+}$like rule & $M D D R^{\ddagger}$ like rule & Rule of five & $W D I^{\S}$ like rule \\
\hline ODN & Qualified & Mid-structure & Suitable & Out of $90 \%$ cutoff \\
\hline ODN1 & Qualified & Mid-structure & Suitable & Out of $90 \%$ cutoff \\
\hline ODN2 & Not qualified & Mid-structure & Suitable & Out of $90 \%$ cutoff \\
\hline ODN3 & Qualified & Mid-structure & Suitable & Out of $90 \%$ cutoff \\
\hline ODN4 & Qualified & Mid-structure & Suitable & Out of $90 \%$ cutoff \\
\hline ODN5 & Qualified & Mid-structure & Suitable & Out of $90 \%$ cutoff \\
\hline ODN6 & Qualified & Mid-structure & Suitable & Out of $90 \%$ cutoff \\
\hline ODN7 & Qualified & Mid-structure & Suitable & Out of $90 \%$ cutoff \\
\hline ODN8 & Qualified & Mid-structure & Suitable & Out of $90 \%$ cutoff \\
\hline ODN9 & Qualified & Mid-structure & Suitable & Out of $90 \%$ cutoff \\
\hline ODN10 & Qualified & Mid-structure & Suitable & Out of $90 \%$ cutoff \\
\hline ODN11 & Qualified & Mid-structure & Suitable & Out of $90 \%$ cutoff \\
\hline ODN12 & Qualified & Mid-structure & Suitable & Out of $90 \%$ cutoff \\
\hline ODN13 & Not qualified & Mid-structure & Suitable & Out of $90 \%$ cutoff \\
\hline ODN14 & Qualified & Mid-structure & Suitable & Out of $90 \%$ cutoff \\
\hline ODN15 & Qualified & Mid-structure & Suitable & Out of $90 \%$ cutoff \\
\hline ODN16 & Qualified & Mid-structure & Suitable & Out of $90 \%$ cutoff \\
\hline ODN17 & Qualified & Mid-structure & Suitable & Out of $90 \%$ cutoff \\
\hline ODN18 & Qualified & Mid-structure & Suitable & Out of $90 \%$ cutoff \\
\hline ODN19 & Qualified & Mid-structure & Suitable & Out of $90 \%$ cutoff \\
\hline ODN20 & Qualified & Mid-structure & Suitable & Out of $90 \%$ cutoff \\
\hline ODN21 & Qualified & Mid-structure & Suitable & Out of $90 \%$ cutoff \\
\hline ODN22 & Qualified & Mid-structure & Suitable & Out of $90 \%$ cutoff \\
\hline ODN23 & Qualified & Mid-structure & Suitable & Out of $90 \%$ cutoff \\
\hline ODN24 & Qualified & Mid-structure & Suitable & Out of $90 \%$ cutoff \\
\hline ODN25 & Qualified & Mid-structure & Suitable & Out of $90 \%$ cutoff \\
\hline ODN26 & Qualified & Mid-structure & Suitable & Out of $90 \%$ cutoff \\
\hline ODN27 & Qualified & Mid-structure & Suitable & Out of $90 \%$ cutoff \\
\hline ODN28 & Qualified & Mid-structure & Suitable & Out of $90 \%$ cutoff \\
\hline ODN29 & Qualified & Mid-structure & Suitable & Out of $90 \%$ cutoff \\
\hline ODN30 & Qualified & Mid-structure & Suitable & Out of $90 \%$ cutoff \\
\hline ODN31 & Qualified & Mid-structure & Suitable & Out of $90 \%$ cutoff \\
\hline ODN32 & Qualified & Mid-structure & Suitable & Out of $90 \%$ cutoff \\
\hline ODN33 & Qualified & Mid-structure & Suitable & Out of $90 \%$ cutoff \\
\hline ODN34 & Qualified & Mid-structure & Suitable & Out of $90 \%$ cutoff \\
\hline ODN35 & Qualified & Mid-structure & Suitable & Out of $90 \%$ cutoff \\
\hline ODN36 & Qualified & Mid-structure & Suitable & Out of $90 \%$ cutoff \\
\hline ODN37 & Qualified & Mid-structure & Suitable & In $90 \%$ cutoff \\
\hline ODN38 & Qualified & Mid-structure & Suitable & Out of $90 \%$ cutoff \\
\hline ODN39 & Qualified & Mid-structure & Suitable & Out of $90 \%$ cutoff \\
\hline
\end{tabular}

${ }^{\dagger}$ Comprehensive medicinal chemistry

${ }^{\ddagger}$ Molecular Detection of Drug Resistance

$\S$ World drug index

reported that the molecule with low toxicity showed Explorer was used to perform the toxicity prediction of a high order of the therapeutic index. OSIRIS Property all the derived compounds. The toxicity parameters were 
Subhajit Sarkar et al.

Table 5: Molecular descriptor properties of oroidin and derived compounds.

\begin{tabular}{|c|c|c|c|c|c|c|c|c|}
\hline Compounds & $m i \log P$ & $T P S A^{a}$ & natoms $^{b}$ & $n O N^{c}$ & $n O H N H^{d}$ & nviolations $^{e}$ & $n r o t b^{f}$ & volume \\
\hline ODN & 2.16 & 99.59 & 19 & 6 & 5 & 0 & 4 & 243.93 \\
\hline ODN1 & 1.68 & 125.62 & 20 & 7 & 7 & 1 & 4 & 255.22 \\
\hline ODN2 & 0.76 & 151.64 & 21 & 8 & 9 & 1 & 4 & 266.51 \\
\hline ODN3 & 0.83 & 142.69 & 22 & 8 & 7 & 1 & 5 & 274.20 \\
\hline ODN4 & -0.76 & 185.78 & 25 & 10 & 9 & 1 & 6 & 304.48 \\
\hline ODN5 & 1.82 & 119.82 & 20 & 7 & 6 & 1 & 4 & 251.95 \\
\hline ODN6 & 2.26 & 140.05 & 21 & 8 & 7 & 1 & 4 & 259.97 \\
\hline ODN7 & 1.34 & 136.89 & 22 & 8 & 6 & 1 & 5 & 270.93 \\
\hline ODN8 & 0.27 & 174.19 & 25 & 10 & 7 & 1 & 6 & 297.93 \\
\hline ODN9 & 2.19 & 99.59 & 20 & 6 & 5 & 0 & 4 & 248.86 \\
\hline ODN10 & 3.02 & 99.59 & 23 & 6 & 5 & 0 & 5 & 275.23 \\
\hline ODN11 & 2.05 & 145.42 & 22 & 9 & 5 & 0 & 5 & 267.26 \\
\hline ODN12 & 0.16 & 157.12 & 22 & 9 & 7 & 1 & 5 & 270.43 \\
\hline ODN13 & -1.12 & 214.65 & 26 & 12 & 9 & 2 & 6 & 314.84 \\
\hline ODN14 & 1.88 & 123.39 & 21 & 7 & 5 & 0 & 4 & 260.79 \\
\hline ODN15 & 2.13 & 99.59 & 20 & 6 & 5 & 0 & 4 & 248.86 \\
\hline ODN16 & 2.77 & 99.59 & 23 & 6 & 5 & 0 & 5 & 275.23 \\
\hline ODN17 & 0.17 & 142.69 & 21 & 8 & 7 & 1 & 5 & 256.32 \\
\hline ODN18 & -0.71 & 159.76 & 23 & 9 & 7 & 1 & 6 & 275.30 \\
\hline ODN19 & 1.13 & 119.82 & 19 & 7 & 6 & 1 & 4 & 234.06 \\
\hline ODN20 & 0.10 & 140.05 & 19 & 8 & 7 & 1 & 4 & 224.20 \\
\hline ODN21 & 1.41 & 108.83 & 20 & 7 & 5 & 0 & 5 & 251.59 \\
\hline ODN22 & 0.40 & 118.06 & 22 & 8 & 5 & 0 & 6 & 277.14 \\
\hline ODN23 & 0.92 & 136.89 & 21 & 8 & 6 & 1 & 5 & 253.05 \\
\hline ODN24 & 0.80 & 148.17 & 23 & 9 & 5 & 0 & 6 & 268.76 \\
\hline ODN25 & 1.51 & 99.59 & 19 & 6 & 5 & 0 & 4 & 230.98 \\
\hline ODN26 & 1.31 & 145.42 & 21 & 9 & 5 & 0 & 5 & 249.38 \\
\hline ODN27 & 2.16 & 99.59 & 19 & 6 & 5 & 0 & 4 & 243.93 \\
\hline ODN28 & 1.13 & 119.82 & 19 & 7 & 6 & 1 & 4 & 234.06 \\
\hline ODN29 & 1.41 & 108.83 & 20 & 7 & 5 & 0 & 5 & 251.59 \\
\hline ODN30 & 1.76 & 125.62 & 20 & 7 & 7 & 1 & 4 & 255.22 \\
\hline ODN31 & 1.43 & 145.85 & 21 & 8 & 8 & 1 & 4 & 263.24 \\
\hline ODN32 & 0.44 & 151.64 & 20 & 8 & 9 & 1 & 4 & 248.62 \\
\hline ODN33 & 2.06 & 102.75 & 19 & 6 & 6 & 1 & 5 & 249.79 \\
\hline ODN34 & 1.95 & 119.82 & 21 & 7 & 6 & 1 & 6 & 268.78 \\
\hline ODN35 & 2.68 & 91.76 & 20 & 6 & 5 & 0 & 6 & 267.32 \\
\hline ODN36 & 1.44 & 125.62 & 21 & 7 & 7 & 1 & 6 & 272.05 \\
\hline ODN37 & 1.63 & 123.39 & 21 & 7 & 5 & 0 & 4 & 260.79 \\
\hline ODN38 & 3.63 & 82.52 & 22 & 5 & 5 & 0 & 6 & 273.07 \\
\hline ODN39 & 0.70 & 157.12 & 24 & 9 & 7 & 1 & 6 & 295.78 \\
\hline
\end{tabular}

${ }^{\text {a Topological polar surface area }}$

${ }^{\mathrm{b}}$ Number of heavy atoms present in the compound

${ }^{c}$ Number of hydrogen bond acceptors

${ }^{\mathrm{d}}$ Number of hydrogen bond donors

${ }^{\mathrm{e}}$ Number of violations made by proposed drug

${ }^{\mathrm{f}}$ Number of rotatable bonds 
Drug Efficacy of Oroidin Derivatives as Hsp90 Inhibitors

Table 6: Pre-ADMET prediction of oroidin and derived compounds

\begin{tabular}{|c|c|c|c|c|c|c|}
\hline Compound & $H I A^{a} \%$ & $\operatorname{Caco}^{b}(\mathrm{~nm} / \mathrm{sec})$ & $M D C K^{c}(\mathrm{~nm} / \mathrm{sec})$ & In vitro $P P B^{d} \%$ & $B B B^{e}$ & Skin permeability \\
\hline ODN & 86.55 & 11.15 & 0.34 & 41.90 & 0.27 & -4.60 \\
\hline ODN1 & 79.84 & 9.56 & 0.47 & 20.86 & 0.07 & -4.81 \\
\hline ODN2 & 67.99 & 13.03 & 0.51 & 17.57 & 0.04 & -4.96 \\
\hline ODN3 & 74.33 & 10.89 & 0.48 & 23.86 & 0.06 & -4.81 \\
\hline ODN4 & 48.96 & 14.88 & 0.51 & 31.41 & 0.04 & -4.92 \\
\hline ODN5 & 80.40 & 10.52 & 0.44 & 39.71 & 0.12 & -4.79 \\
\hline ODN6 & 69.34 & 13.19 & 0.51 & 31.85 & 0.07 & -4.91 \\
\hline ODN7 & 74.64 & 10.23 & 0.45 & 48.50 & 0.07 & -4.81 \\
\hline ODN8 & 51.84 & 14.12 & 0.49 & 58.07 & 0.05 & -4.89 \\
\hline ODN9 & 86.56 & 11.35 & 0.39 & 60.84 & 0.25 & -4.81 \\
\hline ODN10 & 87.10 & 9.94 & 0.36 & 88.02 & 0.60 & -3.70 \\
\hline ODN11 & 69.58 & 11.90 & 0.43 & 64.72 & 0.09 & -4.62 \\
\hline ODN12 & 36.36 & 0.93 & 0.27 & 61.76 & 0.03 & -4.43 \\
\hline ODN13 & 4.46 & 0.61 & 0.43 & 71.40 & 0.03 & -3.63 \\
\hline ODN14 & 81.49 & 8.03 & 0.40 & 48.85 & 0.12 & -4.57 \\
\hline ODN15 & 86.56 & 12.08 & 0.37 & 48.01 & 0.27 & -4.79 \\
\hline ODN16 & 87.10 & 14.99 & 0.34 & 88.52 & 0.94 & -3.60 \\
\hline ODN17 & 55.87 & 17.20 & 0.34 & 18.61 & 0.05 & -4.90 \\
\hline ODN18 & 45.19 & 17.49 & 0.20 & 27.17 & 0.04 & -4.94 \\
\hline ODN19 & 66.10 & 15.36 & 0.39 & 24.57 & 0.12 & -4.80 \\
\hline ODN20 & 18.43 & 6.26 & 1.85 & 32.03 & 0.06 & -4.95 \\
\hline ODN21 & 75.64 & 14.89 & 0.32 & 24.41 & 0.13 & -4.84 \\
\hline ODN22 & 72.09 & 15.21 & 0.44 & 26.61 & 0.08 & -4.99 \\
\hline ODN23 & 56.49 & 16.93 & 0.26 & 32.76 & 0.06 & -4.89 \\
\hline ODN24 & 47.25 & 17.04 & 0.07 & 66.10 & 0.05 & -4.89 \\
\hline ODN25 & 78.91 & 15.11 & 0.27 & 33.94 & 0.18 & -4.86 \\
\hline ODN26 & 48.72 & 17.09 & 0.26 & 50.51 & 0.05 & -4.72 \\
\hline ODN27 & 86.55 & 11.15 & 0.27 & 48.20 & 0.37 & -4.59 \\
\hline ODN28 & 66.11 & 16.34 & 0.20 & 23.11 & 0.12 & -4.84 \\
\hline ODN29 & 75.64 & 14.89 & 0.18 & 23.66 & 0.12 & -4.83 \\
\hline ODN30 & 80.16 & 14.08 & 0.13 & 30.77 & 0.13 & -4.63 \\
\hline ODN31 & 69.24 & 13.73 & 0.25 & 34.45 & 0.07 & -4.81 \\
\hline ODN32 & 47.39 & 17.99 & 0.20 & 13.37 & 0.05 & -4.87 \\
\hline ODN33 & 83.92 & 11.34 & 0.34 & 15.41 & 0.19 & -4.53 \\
\hline ODN34 & 79.01 & 13.35 & 0.43 & 64.49 & 0.11 & -4.54 \\
\hline ODN35 & 87.34 & 12.45 & 0.31 & 26.51 & 0.34 & -4.49 \\
\hline ODN36 & 79.11 & 13.84 & 0.47 & 40.95 & 0.09 & -4.53 \\
\hline ODN37 & 81.49 & 13.67 & 0.39 & 50.99 & 0.15 & -4.56 \\
\hline ODN38 & 88.97 & 15.71 & 0.30 & 94.56 & 1.38 & -3.19 \\
\hline ODN39 & 62.06 & 12.97 & 0.50 & 43.92 & 0.06 & -4.81 \\
\hline
\end{tabular}

${ }^{a}$ Human intestinal absorption

${ }^{\mathrm{b}}$ Human colon carcinoma cell line

${ }^{c}$ Madin-Darby canine kidney

${ }^{\mathrm{d}}$ Plasma protein binding

e Blood brain barrier 
Subhajit Sarkar et al.

Table 7: Drug-likeness of oroidin and derived compounds using OSIRIS

\begin{tabular}{|c|c|c|c|c|c|c|}
\hline Compounds & $\operatorname{clog} P$ & Solubility & Molecular weight (MW) & $\mathrm{TPSA}^{\dagger}$ & Drug-likeness & Drug-score \\
\hline ODN & 1.51 & -3.13 & 387 & 99.59 & -2.24 & 0.45 \\
\hline ODN1 & 0.94 & -3.13 & 402 & 125.6 & -1.93 & 0.46 \\
\hline ODN2 & -0.16 & -2.74 & 417 & 151.6 & -1.93 & 0.46 \\
\hline ODN3 & 0.44 & -2.80 & 430 & 142.6 & -0.63 & 0.54 \\
\hline ODN4 & -1.01 & -2.08 & 473 & 185.7 & -0.63 & 0.52 \\
\hline ODN5 & 1.34 & -3.06 & 403 & 119.8 & -1.72 & 0.47 \\
\hline ODN6 & 0.64 & -2.59 & 419 & 140.0 & -1.81 & 0.47 \\
\hline ODN7 & 0.67 & -2.72 & 431 & 136.8 & -1.21 & 0.49 \\
\hline ODN8 & -0.39 & -1.93 & 475 & 174.1 & -1.21 & 0.38 \\
\hline ODN9 & 2.04 & -3.64 & 405 & 99.59 & -3.61 & 0.39 \\
\hline ODN10 & 2.60 & -3.99 & 455 & 99.59 & -8.83 & 0.34 \\
\hline ODN11 & 0.57 & -3.48 & 432 & 145.4 & -7.33 & 0.38 \\
\hline ODN12 & -3.13 & -0.69 & 389 & 166.9 & -10.30 & 0.44 \\
\hline ODN13 & -6.92 & 0.33 & 469 & 234.2 & -10.32 & 0.40 \\
\hline ODN14 & 1.36 & -3.40 & 412 & 123.3 & -6.05 & 0.39 \\
\hline ODN15 & 1.5 & -3.24 & 405 & 99.59 & -3.61 & 0.41 \\
\hline ODN16 & 2.21 & -3.59 & 455 & 99.59 & -9.27 & 0.36 \\
\hline ODN17 & -0.13 & -2.38 & 352 & 142.6 & 1.06 & 0.78 \\
\hline ODN18 & -0.84 & -1.41 & 380 & 159.7 & 1.30 & 0.80 \\
\hline ODN19 & 0.69 & -2.76 & 325 & 119.8 & -1.96 & 0.50 \\
\hline ODN20 & -0.38 & -1.63 & 263 & 140.0 & 3.32 & 0.94 \\
\hline ODN21 & 0.71 & -2.31 & 339 & 108.8 & -0.16 & 0.66 \\
\hline ODN22 & 0.97 & -2.37 & 369 & 118.0 & -0.21 & 0.63 \\
\hline ODN23 & 0.10 & -2.31 & 353 & 136.8 & 0.49 & 0.72 \\
\hline ODN24 & -0.22 & -1.25 & 382 & 148.1 & 0.69 & 0.74 \\
\hline ODN25 & 0.88 & -2.61 & 327 & 99.59 & -1.51 & 0.53 \\
\hline ODN26 & -0.31 & -2.75 & 354 & 145.4 & -5.22 & 0.44 \\
\hline ODN27 & 1.51 & -3.13 & 387 & 99.59 & -2.41 & 0.45 \\
\hline ODN28 & 0.44 & -2.00 & 325 & 119.8 & -0.25 & 0.66 \\
\hline ODN29 & 0.71 & -2.31 & 339 & 108.8 & -0.33 & 0.64 \\
\hline ODN30 & 1.18 & -3.73 & 402 & 125.6 & -1.96 & 0.44 \\
\hline ODN31 & 1.02 & -3.66 & 418 & 145.8 & -1.45 & 0.46 \\
\hline ODN32 & -0.22 & -2.97 & 339 & 151.6 & -1.08 & 0.55 \\
\hline ODN33 & 1.32 & -2.68 & 389 & 102.7 & -3.48 & 0.44 \\
\hline ODN34 & -1.13 & -2.54 & 417 & 119.8 & -2.97 & 0.44 \\
\hline ODN35 & 1.75 & -2.81 & 403 & 91.75 & -3.50 & 0.42 \\
\hline ODN36 & 0.04 & -2.62 & 416 & 125.6 & -1.87 & 0.47 \\
\hline ODN37 & 0.97 & -3.00 & 412 & 123.3 & -6.49 & 0.41 \\
\hline ODN38 & 2.20 & -3.81 & 441 & 82.5 & -10.92 & 0.36 \\
\hline ODN39 & 0.10 & -2.60 & 461 & 157.1 & -0.71 & 0.51 \\
\hline
\end{tabular}

${ }^{\dagger}$ Topological polar surface area

displayed in OSIRIS as color codes where green indicated a low toxicity. Amino acid residues that interacted with for low, yellow for mediocre, and red for high toxicity. corresponding derived molecules, participated in proteinTable (8) showed that all but merely two compounds had ligand interaction into the binding site of the receptor and 
Table 8: Toxicity prediction of oroidin and derived compounds using OSIRIS

\begin{tabular}{|c|c|c|c|c|}
\hline Compounds & Mutagenic & Tumorigenic & Irritant & $\begin{array}{l}\text { Reproductive } \\
\text { affective }\end{array}$ \\
\hline ODN & Green & Green & Green & Green \\
\hline ODN1 & Green & Green & Green & Green \\
\hline ODN2 & Green & Green & Green & Green \\
\hline ODN3 & Green & Green & Green & Green \\
\hline ODN4 & Green & Green & Green & Green \\
\hline ODN5 & Green & Green & Green & Green \\
\hline ODN6 & Green & Green & Green & Green \\
\hline ODN7 & Green & Green & Green & Green \\
\hline ODN8 & Green & Red & Green & Green \\
\hline ODN9 & Green & Green & Green & Green \\
\hline ODN10 & Green & Green & Green & Green \\
\hline ODN11 & Green & Green & Green & Green \\
\hline ODN12 & Green & Green & Green & Green \\
\hline ODN13 & Green & Green & Green & Green \\
\hline ODN14 & Green & Green & Green & Green \\
\hline ODN15 & Green & Green & Green & Green \\
\hline ODN16 & Green & Green & Green & Green \\
\hline ODN17 & Green & Green & Green & Green \\
\hline ODN18 & Green & Green & Red & Green \\
\hline ODN19 & Green & Green & Green & Green \\
\hline ODN20 & Green & Green & Green & Green \\
\hline ODN21 & Green & Green & Green & Green \\
\hline ODN22 & Green & Green & Green & Green \\
\hline ODN23 & Green & Green & Green & Green \\
\hline ODN24 & Green & Green & Green & Green \\
\hline ODN25 & Green & Green & Green & Green \\
\hline ODN26 & Green & Green & Green & Green \\
\hline ODN27 & Green & Green & Green & Green \\
\hline ODN28 & Green & Green & Green & Green \\
\hline ODN29 & Green & Green & Green & Green \\
\hline ODN30 & Green & Green & Green & Green \\
\hline ODN31 & Green & Green & Green & Green \\
\hline ODN32 & Green & Green & Green & Green \\
\hline ODN33 & Green & Green & Green & Green \\
\hline ODN34 & Green & Green & Green & Green \\
\hline ODN35 & Green & Green & Green & Green \\
\hline ODN36 & Green & Green & Green & Green \\
\hline ODN37 & Green & Green & Green & Green \\
\hline ODN38 & Green & Green & Green & Green \\
\hline ODN39 & Green & Green & Green & Green \\
\hline
\end{tabular}

the number of hydrogen bonds formed during interactions were enlisted in Table 9.

\section{DISCUSSION}

\section{HOMO-LUMO Analysis}

A compound's reactivity depends upon the difference of energy between the HOMO and LUMO of a system. ${ }^{[46]}$ HOMO-LUMO energies were calculated by DFT-UB3LYP using the basis set $6-311++\mathrm{G}(\mathrm{d}, \mathrm{p})$ and the contour diagrams were displayed in Fig. 3 where positive and negative lobes were represented by red and green color, respectively. Homo has a tendency to give away its electron and behaves as nucleophile whereas LUMO, due to its electron-accepting tendency, behaves as an electrophile. ${ }^{[47]}$

The knowledge of HOMO-LUMO band gap energy $\left(\Delta \mathrm{E}_{\mathrm{HL}}\right)$ is useful in determining various molecular properties like chemical reactivity, kinetic stability, optical polarizability, and chemical hardness-softness of molecules. The smaller the $\Delta \mathrm{E}_{\mathrm{HL}}$ value, the greater the chemical reactivity and polarizability, which corresponds to less stability. ${ }^{[48,49]}$ On the contrary higher, the value of $\Delta \mathrm{E}_{\mathrm{HL}}$ points out greater chemical stability and low reactivity. Moreover, molecules that possess a higher dipole moment show a tendency towards strong intermolecular interaction. The degree of chemical reactivity determines the nature of chemical hardness and softness. ${ }^{[50]}$
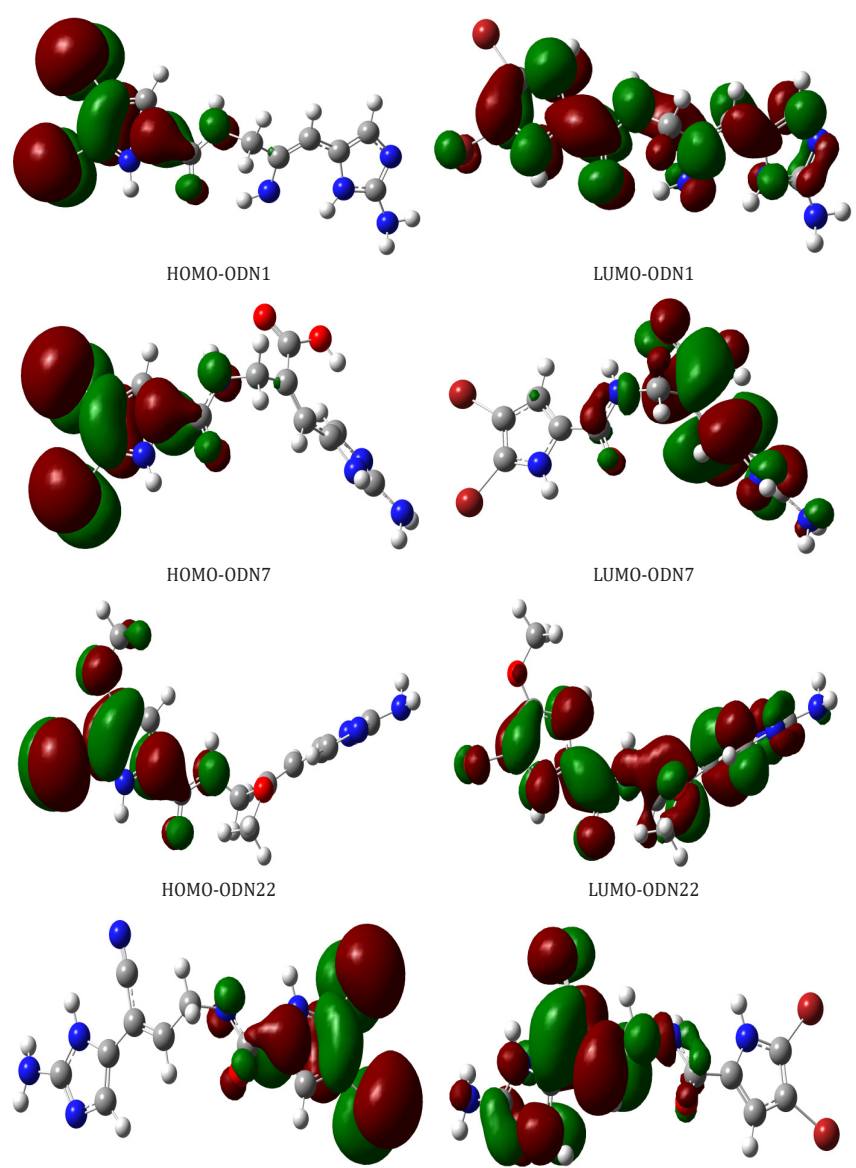

HOMO-ODN37

LUMO-ODN37

Fig 3: Contours of the occupied and unoccupied molecular orbitals of the ligands. 
Subhajit Sarkar et al.

Table 9: List of amino acids of oroidin and derived compounds, participating in protein-ligand interaction into binding site, with receptor and the number of hydrogen bonds.

\begin{tabular}{|c|c|c|c|}
\hline Compounds & $\Delta G_{\text {Binding }}(\mathrm{kcal} / \mathrm{mol})$ & Key protein-ligand interaction & H-bond \\
\hline ODN & -11.31 & $\begin{array}{l}\text { Asn51, Leu107, Gly108, Phe138, Tyr139, Ala111, Val136, Ile26, Gln23, Phe22, Ala21, } \\
\text { Phe170, Ile104, Leu103, Trp162 }\end{array}$ & 5 \\
\hline ODN1 & -12.07 & $\begin{array}{l}\text { Asn105, Ile104, Leu103,Gln23,Ala21, Phe170, Phe22, Trp162, Thr109, Leu107, Gly108, } \\
\text { Ile26, Tyr139, Phe138, Val150 }\end{array}$ & 3 \\
\hline ODN2 & -11.94 & $\begin{array}{l}\text { Phe138, Tyr 139, Leu103, Trp162, Phe22, Phe170, Ile104, Gln23, Ile26, Gly108, Leu107, } \\
\text { Ala111, Val136, Ala21 }\end{array}$ & 4 \\
\hline ODN3 & -12.63 & $\begin{array}{l}\text { Asn105, Ile104, Leu103, Met98, Val150, Trp162, Leu107, Phe170, Gly108, Thr109, Ala21, } \\
\text { Gln23, Phe22, Ile25, Tyr138, Phe138 }\end{array}$ & 3 \\
\hline ODN4 & -12.33 & $\begin{array}{l}\text { Leu48, Asn51, Thr184, Phe138, Tyr139, Val150, Trp162, Phe22, Phe170, Leu103, } \\
\text { Leu107, Met98, Gly97, Ala55 }\end{array}$ & 4 \\
\hline ODN5 & -12.54 & $\begin{array}{l}\text { Val150, Trp162, Leu103, Phe138, Met98, Tyr139, Leu107, Phe170, Ile104, Phe22, Phe20, } \\
\text { Ala21, Gln23, Asn105, Ile26, Gly108 }\end{array}$ & 4 \\
\hline ODN6 & -10.97 & $\begin{array}{l}\text { Asn51, Met98, Phe138, Leu107, Tyr139, Ala111, Val136, Gly135, Leu103, Trp162, Ile104, } \\
\text { Phe170, Phe22, }\end{array}$ & 5 \\
\hline ODN7 & -12.60 & $\begin{array}{l}\text { Asn105, Ile104, Phe170, Phe22, Ile26, Gly108, Thr109, Gln23, Leu103, Trp162,Phe138, } \\
\text { Asn51, Val136, Tyr139 }\end{array}$ & 5 \\
\hline ODN8 & -11.04 & $\begin{array}{l}\text { Ala111, Leu107, Met98, Tyr139, Val136, Gly135, Gly137, Phe138, Thr184, Val186, Ala55, } \\
\text { Asn51, Leu48, Ala52, Asp93, Lys185,Val92, Leu91 }\end{array}$ & 6 \\
\hline ODN9 & -11.26 & $\begin{array}{l}\text { Ala21, Gln23, Phe22, Ile26, Phe170, Trp162, Tyr139, Val150, Phe138, Leu107, Leu103, } \\
\text { Gly108, Thr109, Asn105, Ile104 }\end{array}$ & 2 \\
\hline ODN10 & -9.87 & $\begin{array}{l}\text { Lys185, Val92, Asp93,Thr184, Leu91, Val186, Ala52, Ala55, Met98, Leu48, Asn51, } \\
\text { Phe138, Leu107, Tyr139, Gly137, Val136, Gly135, Ala111 }\end{array}$ & 3 \\
\hline ODN11 & -11.37 & $\begin{array}{l}\text { Leu107, Leu103, Phe138, Trp162, Tyr139, Phe170, Phe22, Gln23, Ile104, Gly108, } \\
\text { Asn105, Thr109, Asn51 }\end{array}$ & 6 \\
\hline ODN12 & -12.34 & $\begin{array}{l}\text { Gly135, Val136, Gly137, Ala111, Ile110, Ile26, Gly108, Gln23, Leu107, Phe138, Tyr139, } \\
\text { Asn51, Phe22, Asn105, Ile104, Phe170, Trp162, Leu103 }\end{array}$ & 8 \\
\hline ODN13 & -11.60 & $\begin{array}{l}\text { Ala55, Ala52, Gly97, Ile49, Asn51, Ile78, Asp93, Met98, Leu48, Thr184, Leu91, Val186, } \\
\text { Val150, Trp162, Leu103, Tyr139, Phe138,Leu107 }\end{array}$ & 5 \\
\hline ODN14 & -10.42 & $\begin{array}{l}\text { Asn51, Ala52, Ala55, Leu48, Leu91, Asp93, Val92, Lys185, Thr184, Val186, Val150, } \\
\text { Trp162, Leu103, Leu107, Tyr139,Phe138,Met98 }\end{array}$ & 4 \\
\hline ODN15 & -11.85 & $\begin{array}{l}\text { Gly135, Val136, Asn51, Tyr139, Phe138, Ile26, Thr109, Gly108, Leu107, Phe22, Trp162, } \\
\text { Asn105, Phe170, Leu103, Ile104, Gln23 }\end{array}$ & 5 \\
\hline ODN16 & -10.17 & $\begin{array}{l}\text { Val136, Gly135, Tyr139, Ala111, Phe138, Phe22, Ile110, Gly108, Phe170, Trp162, } \\
\text { Leu107, Leu103, Asn105, Asp102, Ile104 }\end{array}$ & 3 \\
\hline ODN17 & -12.68 & $\begin{array}{l}\text { Val136, Gly135, Gly137, Ile26, Tyr139, Ala111, Phe138, Gln23, Phe22, Asn51, Gly108, } \\
\text { Ala21, Leu107, Trp162, Phe170, Leu103, Ile104 }\end{array}$ & 8 \\
\hline ODN18 & -13.18 & $\begin{array}{l}\text { Val136, Gly135, Ile26, Tyr139, Ala111, Gln23, Phe22, Phe138, Asn51, Gly108, Ala21, } \\
\text { Thr109, Leu107, Phe170, Trp162, Leu103, Asn105, Ile104 }\end{array}$ & 7 \\
\hline ODN19 & -11.78 & $\begin{array}{l}\text { Val136, Gly135, Ile26, Tyr139, Ala111, Gln23, Phe22, Phe138, Asn51, Gly108, Ala21, } \\
\text { Leu107, Phe170, Leu103, Ile104 }\end{array}$ & 7 \\
\hline ODN20 & -11.53 & $\begin{array}{l}\text { Gly135, Asn51, Gly137, Val136, Phe138, Leu107, Tyr139, Gly108, Ile26, Leu103, Trp162, } \\
\text { Phe22, Gln23, Phe170, Ile104 }\end{array}$ & 6 \\
\hline ODN21 & -11.63 & $\begin{array}{l}\text { Phe138, Tyr139, Leu107, Gly108, Trp162, Leu103, Phe22, Gln23, Phe170, Ile104, } \\
\text { Asn105,Thr109,Asn51, }\end{array}$ & 5 \\
\hline ODN22 & -11.72 & $\begin{array}{l}\text { Asp102, Asn51, Gly135, Leu107, Thr109, Leu103, Val136, Gly108, Phe138, Trp162, } \\
\text { Tyr139,Gln23, Phe170, Phe22,Asn105, Ile104 }\end{array}$ & 8 \\
\hline ODN23 & -12.25 & $\begin{array}{l}\text { Asn51, Leu107, Leu103, Asn105, Gly135, Thr109, Ile104, Gly108, Trp162, Tyr139, } \\
\text { Val136, Phe138, Gly137, Phe170, Gln23, Phe22 }\end{array}$ & 8 \\
\hline ODN24 & -12.23 & $\begin{array}{l}\text { Gly135, Ala111, Val135, Gly108, Leu107, Asn51, Gly137, Gln23, Ile26, Tyr139, Phe138, } \\
\text { Ile104, Leu103, Phe22, Trp162, Phe20, Phe170, Ala21 }\end{array}$ & 6 \\
\hline
\end{tabular}




\begin{tabular}{|c|c|c|c|}
\hline Compounds & $\Delta G_{\text {Binding }}(\mathrm{kcal} / \mathrm{mol})$ & Key protein-ligand interaction & H-bond \\
\hline ODN25 & -11.50 & $\begin{array}{l}\text { Thr109, Asn106, Ala111, Val136, Leu107, Gly108, Asn105, Gln23, Phe138, Tyr139, } \\
\text { Leu103, Ile104, Phe22, Trp162, Phe170 }\end{array}$ & 4 \\
\hline ODN26 & -11.60 & $\begin{array}{l}\text { Asn51, Val136, Ala111, Leu107, Gly108, Phe138, Tyr139, Ile26, Gln23, Asn105, Leu103, } \\
\text { Phe22, Ile104, Trp162, Phe170 }\end{array}$ & 6 \\
\hline ODN27 & -11.56 & $\begin{array}{l}\text { Thr109, Ala111, Asn105, Leu107, Gly108, Gln23, Met98, Ile104, Leu103, Tyr139, Phe22, } \\
\text { Phe138, Phe170, Trp162 }\end{array}$ & 4 \\
\hline ODN28 & -11.28 & $\begin{array}{l}\text { Thr109, Asn105, Leu107, Gly108, Gln23, Asn51, Ile104, Leu103, Tyr139, Phe138, Phe22, } \\
\text { Phe170, Trp162 }\end{array}$ & 6 \\
\hline ODN29 & -12.08 & $\begin{array}{l}\text { Gly108, Val136, Leu107, Gln23, Ile26, Ile104, Tyr139, Phe22, Leu103, Ala21, Phe138, } \\
\text { Phe170, Trp162, Val150 }\end{array}$ & 5 \\
\hline ODN30 & -12.39 & $\begin{array}{l}\text { Thr109, Gln23, Ile26, Gly108, Asn105, Leu107,Ala21, Tyr139, Phe22, Ile104, Phe20, } \\
\text { Phe138, Phe170, Lu103, Met98, Trp162, Val150 }\end{array}$ & 8 \\
\hline ODN31 & -11.83 & $\begin{array}{l}\text { Gln23, Ile26, Ala21, Thr109, Phe22, Gly108, Asn105, Tyr139, Phe170, Ile104, Leu107, } \\
\text { Trp162 }\end{array}$ & 9 \\
\hline ODN32 & -11.66 & $\begin{array}{l}\text { Thr109, Leu107, Met98, Gly108, Tyr139, Ile26, Asn105, Leu103, Gln23, Ile104, Trp162, } \\
\text { Phe22, Phe170, Phe20, Ala21 }\end{array}$ & 7 \\
\hline ODN33 & -10.88 & $\begin{array}{l}\text { Gly108, Leu107,Ile26, Gln23, Tyr139, Phe138, Phe22, Leu103, Val150, Ile104, Trp162, } \\
\text { Phe170, Ala21 }\end{array}$ & 6 \\
\hline ODN34 & -9.91 & $\begin{array}{l}\text { Thr109, Leu107, Gly108, Asp102, Phe138, Tyr139, Gln23, Asn105, Leu103, Phe22, } \\
\text { Ile104, Trp162, Phe170, Ala21 }\end{array}$ & 6 \\
\hline ODN35 & -9.50 & $\begin{array}{l}\text { Asn51, Ile49, Ala52, Ile78, Leu48, Leu107, Asp93, Phe138, Leu91, Met98, Thr184, Val92, } \\
\text { Val186, Leu103, Val150 }\end{array}$ & 2 \\
\hline ODN36 & -11.89 & $\begin{array}{l}\text { Thr109, Ala111, Val136, Leu107, Met98, Phe138, Tyr139, Gly108, Asn105, Gln23, } \\
\text { Leu103, Val150, Trp162, Ile104, Phe22, Phe170 }\end{array}$ & 5 \\
\hline ODN37 & -11.41 & $\begin{array}{l}\text { Thr109, Leu107, Gly108, Met98, Phe138, Tyr139, Ile26, Asn105, Gln23, Leu103, Val150, } \\
\text { Trp162, Ile104, Phe22, Phe170, Ala21 }\end{array}$ & 2 \\
\hline ODN38 & -11.88 & $\begin{array}{l}\text { Asn51, Ala111, Leu107, Phe138, Tyr139, Ile26, Gly108, Gln23, Asn105, Leu103, Phe22, } \\
\text { Val150, Trp162, Ile104, Phe170 }\end{array}$ & 4 \\
\hline ODN39 & -9.88 & $\begin{array}{l}\text { Asp54, Ala55, Ile96, Gly95, Ala52, Gly97, Asp93, Gly183, Asn51, Met98, Thr184, Leu107, } \\
\text { Leu103, Phe138, Val150, Tyr139, Trp162 }\end{array}$ & 4 \\
\hline
\end{tabular}

Amino acids with bold letters are the participators in forming $\mathrm{H}$-bonds.

Here we observed that twenty-eight derived compounds possessed lower $\Delta \mathrm{E}_{\mathrm{HL}}$ value than that of precursor

ODN (4.29 eV) (Table 2). Among all, ODN18 contained the highest energy gap ( $4.59 \mathrm{eV})$ and was considered as least reactive. On the other hand, ODN26 with the lowest value $(3.07 \mathrm{eV})$ was highly polarizable, reactive, and behaved as a soft species. Analyzing the dipole moment values it had been found that ODN12, ODN17, ODN23, and ODN39 derived molecules had a higher tendency to take part in strong intermolecular interaction (Table 2).

\section{Global Reactivity Descriptors}

Several new chemical descriptor parameters have been suggested to understand some important pharmacological properties and the eco-toxicological character of drug molecules. Conceptual DFT based parameters are being used to understand the reactivity of molecules by calculating the chemical potential, global hardness, softness, and electrophilicity index. Ionization potential (I) and electron affinity (A). Expressions of them are as follows:

$$
\begin{aligned}
& I=-E_{\text {Hомо }} \\
& A=-E_{\text {LUMO }}
\end{aligned}
$$

Using the above other descriptor properties such as chemical potential $(\mu)$, global hardness $(\eta)$, global softness $(S)$, electronegativity $(\sigma)$, and electrophilicity index $(\omega)$ can be calculated.

$$
\begin{aligned}
& \mu=\left(E_{\text {LUMO }}+E_{\text {HOMO }}\right) / 2 \\
& \eta=\left(E_{\text {LUMO }}-E_{\text {HOMO }}\right) / 2 \\
& S=1 / \eta \\
& \sigma=-\mu \\
& \omega=\mu^{2} / 2 \eta
\end{aligned}
$$

Global hardness predicts the chemical stability and measures the resistance to change the electronic charge distribution in a molecule. ${ }^{[48,49]}$ Electrophilicity index $(\omega)$ is the measure of electron acceptance capacity of a molecule. ${ }^{[50]}$ The higher value of chemical softness of ODN1, ODN11, and ODN26 suited them to act as a soft molecule with high polarizability while the higher value of global hardness of ODN18, ODN33, and ODN39 were considered as electron-withdrawing species and could be treated as electrophiles (Table 3). 


\section{Toxicity and Molecular Descriptor Properties}

In this study, we tested silico toxicity parameters by using the OSIRIS property explorer that indicates mutagenic, tumorigenic, irritant, and reproductive index of the designed ligands. All the compounds, except ODN8 and ODN18, qualified the above-desired parameters (Table 8). Solubility, drug-likeness and drug-score values were also evaluated from this web application. ${ }^{[51]}$ Molinspiration web toolkit predicted the bioactive scores which help us to screen best inhibitors of Hsp90 and we calculated valuable parameters such that miLogP, topological polar surface area (TPSA), number of rotatable bonds (nrotb), number of hydrogen bond acceptors (nON) and donors (nOHNH). Permeability through cell membrane depends on the miLogP parameter and TPSA provides the tendency of generating hydrogen bonds. The number of rotatable bonds represents the flexibility of the compound. Molecular properties and structural features, irrespective of known drugs were scrutinized based on the drug-likeness data of molecules. ${ }^{[51]}$ The excellent results listed in (Table 5, 7) indicate that most of the derived compounds have good potential to qualify as future drugs.

Rule of five states that compounds having some violations $\leq 1$ shows good bioavailability and the obtained results confirmed that all the derived compounds may behave as good bioavailable drugs. High probability of solubility in cellular membranes requires $\mathrm{nOHNH} \leq 5$ and sixteen of the derived compounds followed this rule. ${ }^{[52]}$ Apart from this, all the derived molecules have a molecular weight (MW) $<500, \mathrm{nON} \leq 10$ (except ODN13), cLogP $\leq 5$ and nrotb $\leq 6$ and qualified oral route of newly designed drugs. ${ }^{[33]}$ Molecules with TPSA $>140$ exhibit poor permeability through the cell membrane and TPSA $<90$ is required to penetrate the blood-brain barrier in the central nervous system. Twenty-one of the derived compounds have the desired TPSA range (90 ̊̊2-140Å2) and could be considered as candidate drugs.

\section{ADME Prediction and Drug-likeness Properties}

The ADME is an abbreviation used in the pharmacokinetics for absorption, distribution, metabolism, and excretion and affects the compound's performance and pharmacological activity as a drug. ${ }^{[54]}$ Most of the derived compounds have shown satisfactory in silico ADME properties (Table 6) Six derived compounds showed better results as intestinal absorption (HIA) than standard ODN closed to $86.55 \%$. ODN38 secured the highest HIA (88.97\%); hence, it showed maximum absorption. All the compounds exhibited low or moderate in vitro Caco2 and MDCK cell permeability. In vivo blood-brain barrier (BBB) penetration discovered that most of the compounds (except ODN38) had no or low $(\mathrm{BBB}<1)$ absorption into the central nervous system (CNS), causing no or less adverse effect towards CNS. Plasma protein binding refers to the degree of binding of a drug to proteins within the blood. The less is the binding of the more efficient drug causes more penetration through the cell membrane. All the compounds recorded $\mathrm{PPB}<90 \%$ (except ODN38), possessing better diffusion into the cell. Skin permeability data indicated that they could have a transdermal application. Moreover, except ODN2 and ODN13 all the derived molecules obeyed CMC, MDDR, and WDI like rules as displayed in Table 4. Based on these observations, we may conclude that most of these derived molecules have the capability of good drug-likeness and ADME properties.

\section{Molecular Docking and Pharmacophore Studies}

Molecular docking is the most sound and significant method to realize the foundation of protein-ligand interaction. ${ }^{[55]}$ We have studied this method to ascertain the binding affinity of the ligands with the active sites of Hsp90 (PDB: 3NMQ). The standard structure of ODN was marked into three subsections, two 5-member rings (A, B) and linker (C) (Fig. 1). Molecular docking was done to demonstrate the recognition mode between derived ODN derivatives with Hsp90 and a graphical illustration of best results displayed in Fig. 4.

Katja-Emilia Lillsunde and his co-workers found that oroidin derivatives (imidazole ring substituted by the thiazole ring isosterically) could be used as potent DNA gyrase inhibitors targeting ATP-binding site of Hsp90 required for viral replication. ${ }^{[12]}$ Based on their experimental work we followed a theoretical approach to design oroidin derivatives based on functionalisation

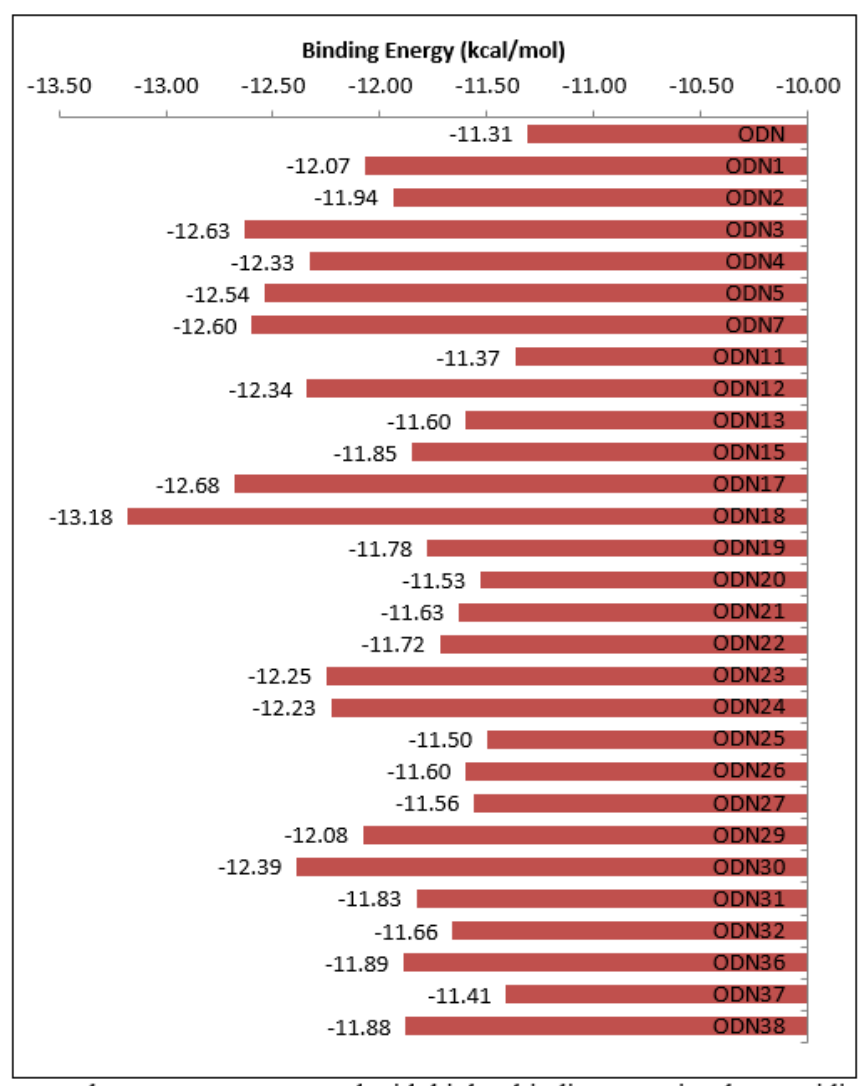

Fig 4: Bar graph represents compound with higher binding energies than oroidin (ODN). 
in the native oroidin compound. We got better binding values when substitution at various positions of oroidin (Fig. 2) was done by amide, amine, phosphate, hydroxyl, fluorine, methoxy, and carboxylic acid groups but the groups like trifluoromethyl, cyanide, and nitro failed to exhibit better result.

The docking results revealed that 28 derived compounds had a better result of binding energy than the standard ODN (-11.31 Kcal/mol) (Table 1). A 2D and 3D ligand-receptor docking interactions of the four best derivatives are shown in Figs. 5 and Fig. 6) while the rest are shown in the supplementary file. The residues of the active site of Hsp90 were found to be Asn51, Leu107, Gly108, Phe138, Tyr139, Ala111, Val136, Ile26, Gln23, Phe22, Ala21, Phe170, Ile104, Leu103 and Trp162 (Fig. 7). From the analysis of the molecular alignment of docked complexes, it was found that all the derived compounds docked at the same region of Hsp90 as in the case of ODN (Fig. 8). Out of them, 5 derivatives indicated considerably higher binding energy $(<-12.5 \mathrm{Kcal} / \mathrm{mol})$, whereas the rest 23 compounds have moderately higher binding energy $(<-11.31 \mathrm{Kcal} / \mathrm{mol})$ compared to precursor ODN.
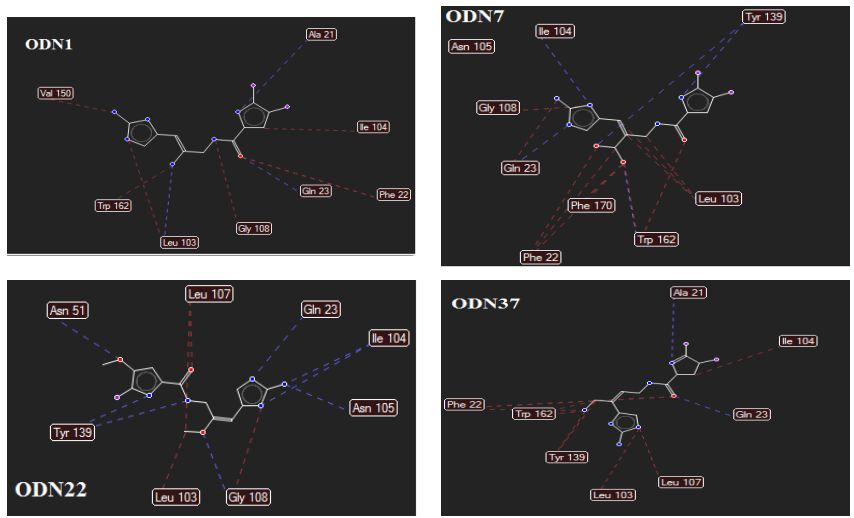

Fig 5: 2-D docking model of the ligands (a) ODN1,

(b) ODN7, (c) ODN22, (d) ODN37 with Hsp90 (PDB: 3NMQ) protein (Hydrogen bonds are represented as blue line and steric interactions are represented as red or no line).

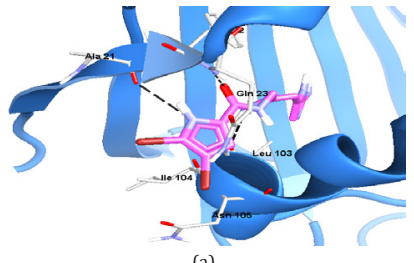

(a)

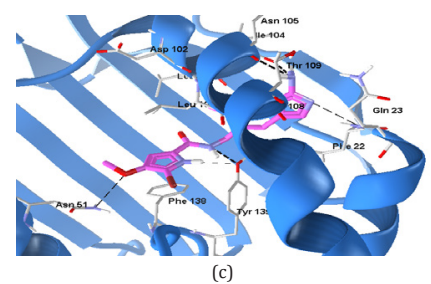

Fig. 6: 3D docking model of ligands (a) ODN1, (b) ODN7, (c) ODN22, (d) ODN37, with Hsp90 (PDB: 3NMQ) protein. (Dark lines indicate the hydrogen bond interaction).
Incorporation of one or more amide groups either at the side ring (B) or at linker (C) as designed in ODN3, ODN17, ODN4, ODN18, resulted in a better binding score. ODN18 achieved maximum binding energy (-13.18 Kcal/ mol) implies maximum stability. Seven strong hydrogen bonds, caused by 2 amide groups incorporated at the side chain of the 5-membered ring (B), have been formed at the binding site. $\mathrm{H}$ bonds were found to have interacted with the Gly135, GIn23, Phe138, Ala21, Ile104 residues (Supplementary Fig. S2), and bond distances lying between $1.97 \AA$ to $3.40 \AA$ (Supplementary Fig. S3). The amino acids Val136, Gly135, Ile26, Tyr139, Ala111, Gln23, Phe22, Phe138, Asn51, Gly108, Ala21, Thr109, Leu107, Phe170, Trp162, Leu103, Asn105, Ile104 were found to be connected (Supplementary Fig. S4) with ODN18 through hydrophobic interaction(Table 9). ODN35 showed minimum stability with the lowest binding score $(-9.50 \mathrm{Kcal} / \mathrm{mol})$ that might be due to the electrostatic repulsion effect of the methoxy group with the receptor moiety.

Mono substituted amine group at linker (C) in ODN1 exhibited better docking value $(-12.07 \mathrm{Kcal} / \mathrm{mol})$ than
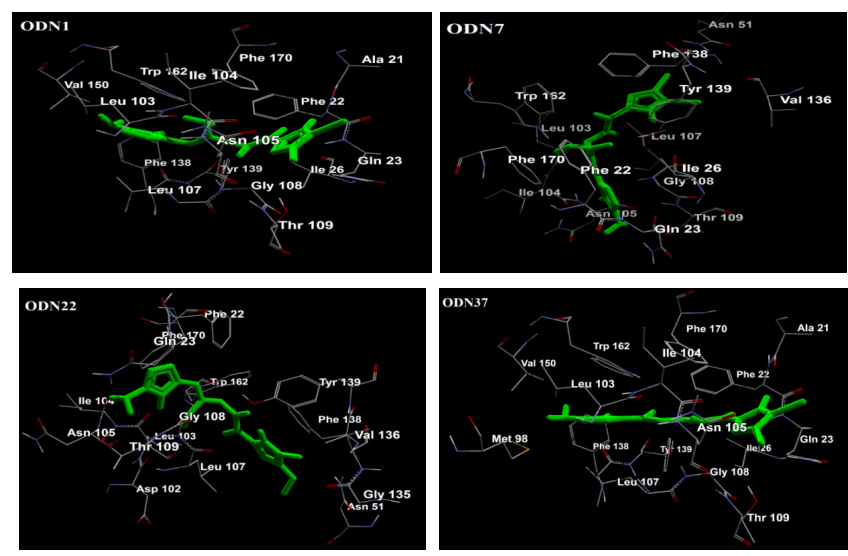

Fig 7: Pose interaction view of of compounds with the target protein Hsp90.

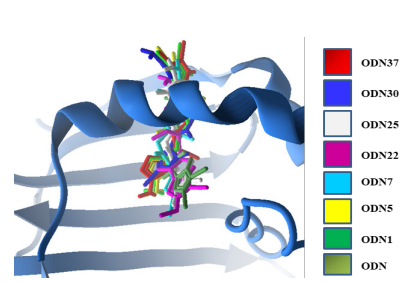

(a)

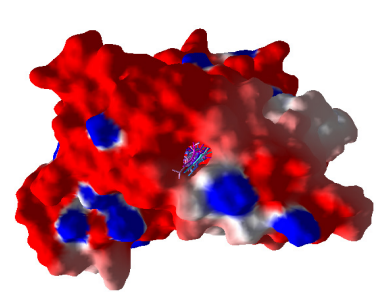

(c)

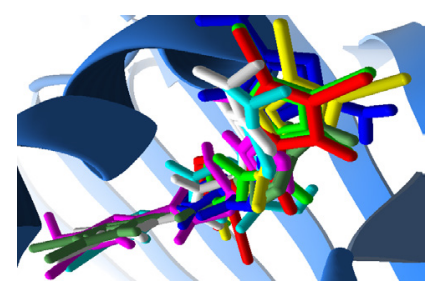

(b)

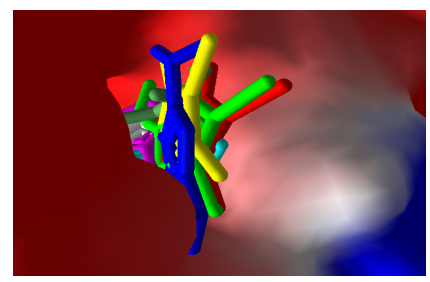

(d)
Fig 8: (a) Docking model of the oroidin and other 7 best ligands with Hsp90 (PDB:3NMQ) protein. (b) Zoomed view of the active site showing the docked molecules. (c) Surface view of the ligands into binding site and (d) zoomed view of the ligands into protein surface. 


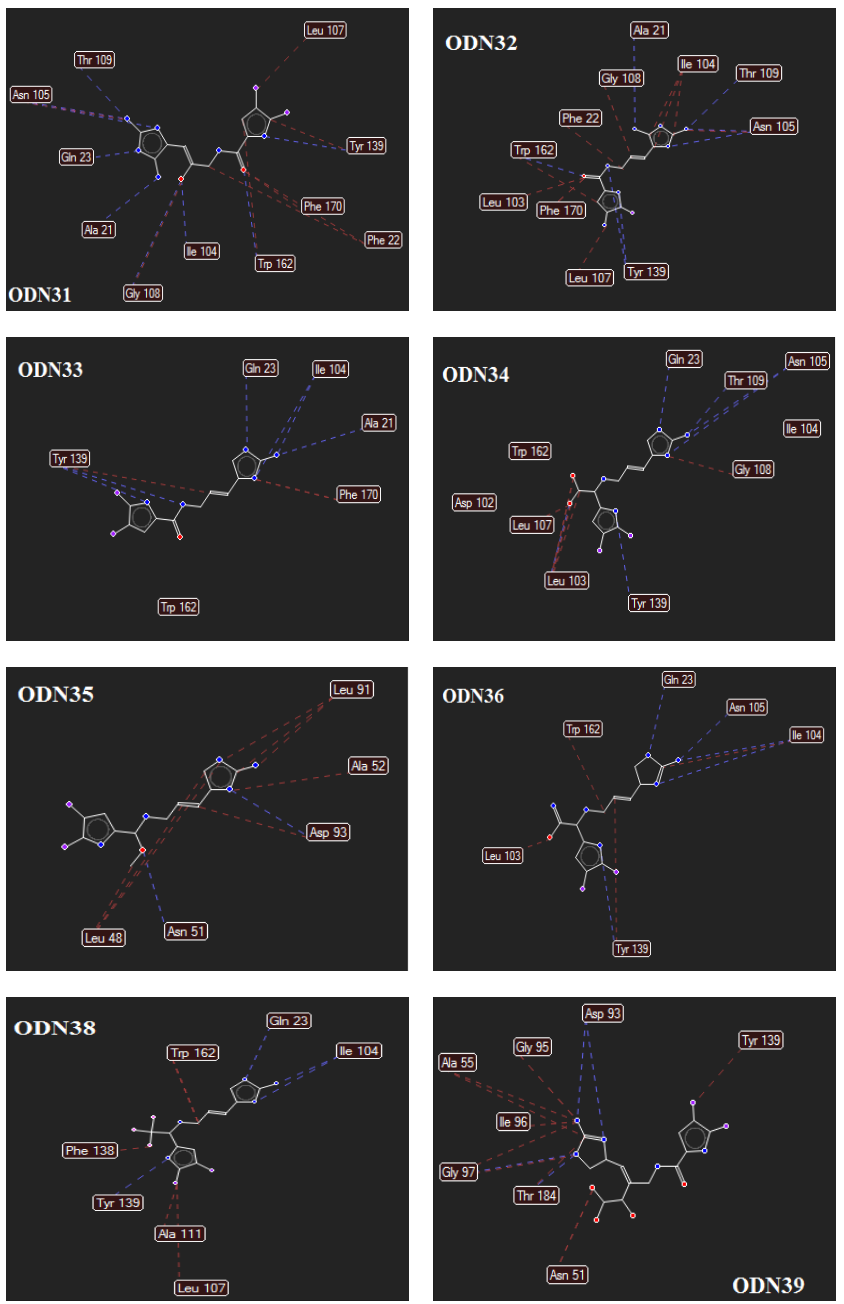

Fig S2: 2-D docking model of the ligands with Hsp90 (PDB: 3NMQ) protein (Hydrogen bonds are represented as blue line and steric interactions are represented as red or no line)

the di-substituted amine groups at the linker in ODN2 $(-11.94 \mathrm{Kcal} / \mathrm{mol})$. Surprisingly better results -12.39 $\mathrm{Kcal} / \mathrm{mol}$ have been found in ODN30 when the position of amine group $\mathrm{C}-2$ at the side ring (A) have been changed to $\mathrm{C}-4$. Strong electrostatic interaction into the binding site is responsible for the better docking results in both cases.

Like amine group, monosubstituted hydroxyl group, found in ODN5 at C-7 and ODN19 at C-12 position displayed better docking result $-12.54 \mathrm{Kcal} / \mathrm{mol}$ and $-11.78 \mathrm{Kcal} / \mathrm{mol}$ respectively than the di-substituted ODN20 (-11.53 Kcal/ mol ) and ODN6 ( $-10.17 \mathrm{Kcal} / \mathrm{mol})$ due to the electrostatic repulsion causes less binding affinity with the binding receptor. Similarly, two large phosphate groups in ODN13, at the side chain of 5-member ring (B) and linker (C) replacing $\mathrm{Br}$ at $\mathrm{C}-13$ and $\mathrm{H}$ at $\mathrm{C}-8$ positions respectively, developed low interaction with the binding site, resulted in lower binding energy $(--11.60 \mathrm{Kcal} / \mathrm{mol})$. But the substitution of one phosphate group at C-13, found in ODN12, favored higher interaction at the binding region
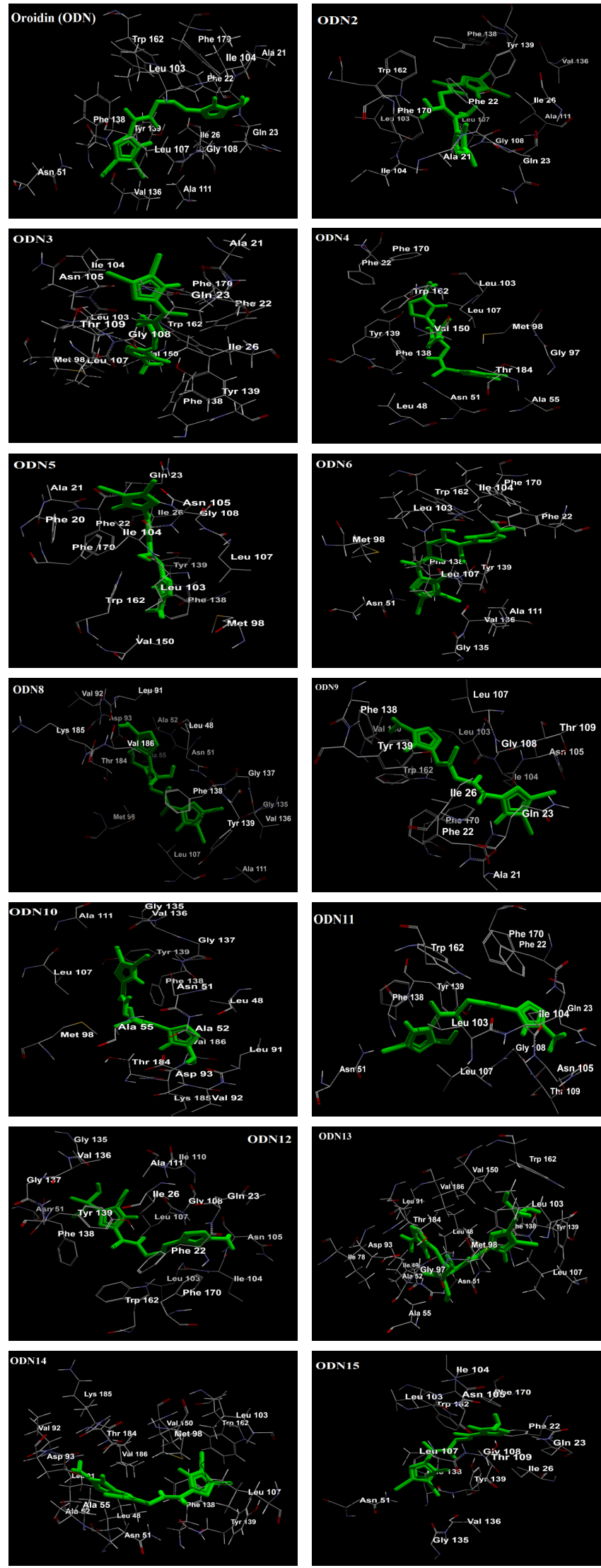

Fig S4: Pose interaction view of compounds with the target protein Hsp90. 

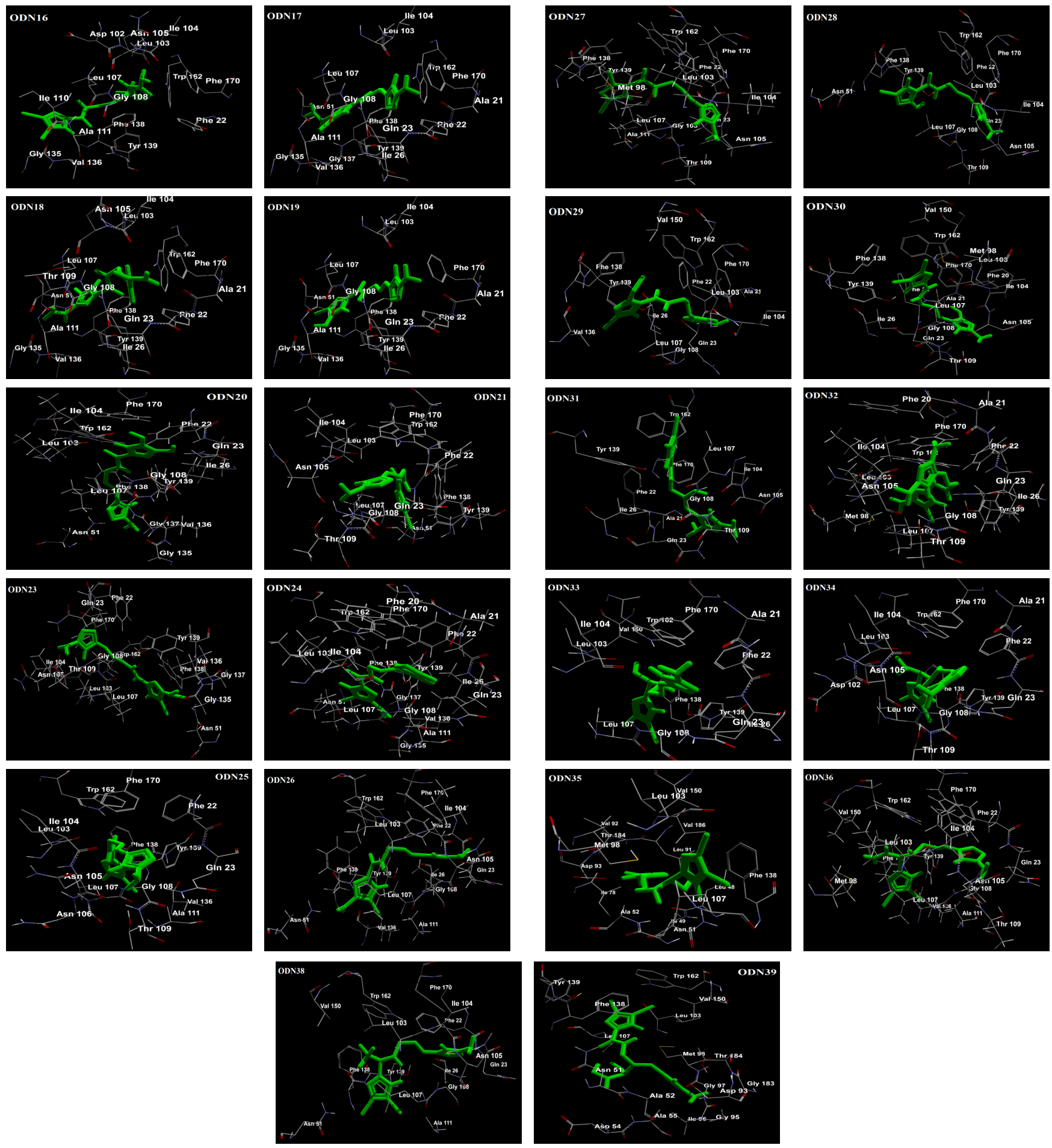

Fig S4: Pose interaction view of compounds with the target protein Hsp90.

and produced higher binding energy $(-12.34 \mathrm{Kcal} / \mathrm{mol})$ than ODN13.

The presence of less polar one or more methoxy groups in ODN29, ODN22, ODN21, indicated moderate binding value, following moderate hydrophobic interaction, lying in the range $-12.08 \mathrm{Kcal} / \mathrm{mol}$ to $-11.63 \mathrm{Kcal} / \mathrm{mol}$. The very poor result we got when -0 at $\mathrm{C}-10$ position was replaced by a methoxy group, found in ODN35. Introduction of one highly polar $-\mathrm{COOH}$ group in place of $-\mathrm{H}$ at the $\mathrm{C}-7$ position in the side chain of linker producing ODN7 and instead of -Br at C-13 position in the side ring (B) producing ODN23, would favor greater electrostatic interaction, Vander Waals interaction, and $\mathrm{H}$-bond interaction into the binding site promoting the excellent value of binding energies of $-12.6 \mathrm{Kcal} / \mathrm{mol}$ and $-12.25 \mathrm{Kcal} / \mathrm{mol}$ respectively. But, the incorporation of second $-\mathrm{COOH}$ group at C-6, found in 

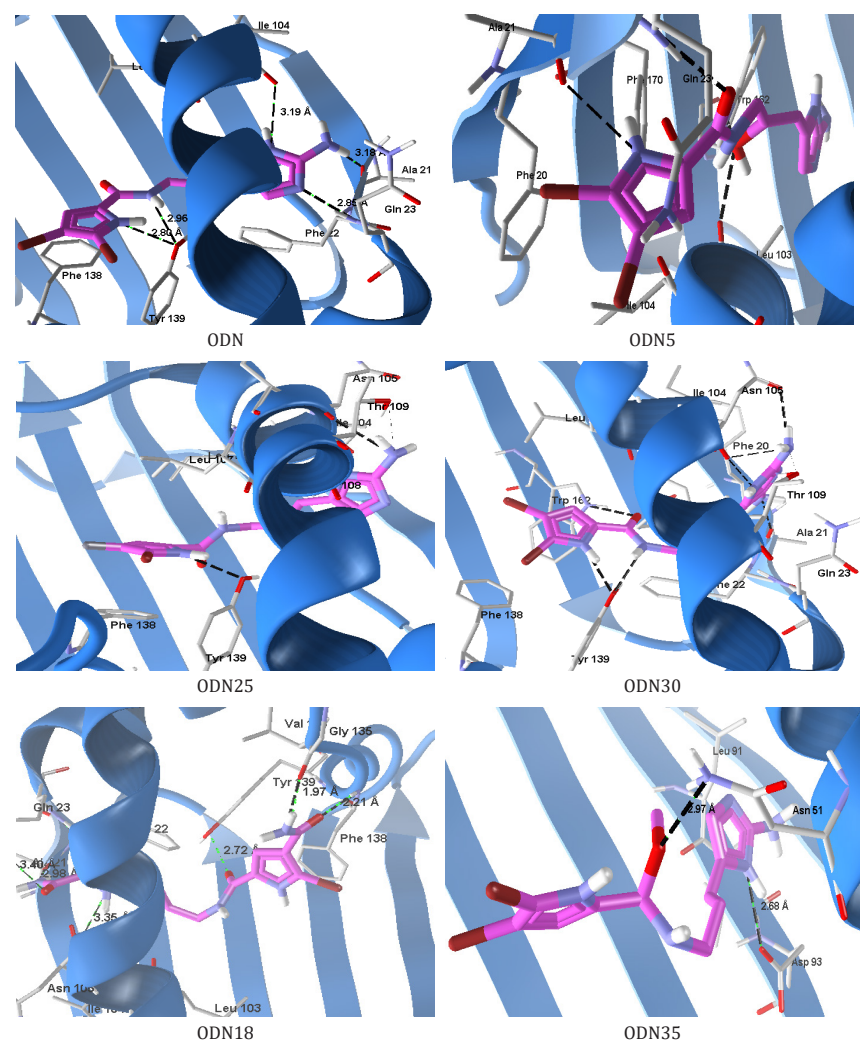

Fig S3: 3D docking model of ligands with Hsp90 (PDB: 3NMQ) protein. (Black lines indicate the hydrogen bond interaction).

ODN8 and replacing -0 at C-10 found in ODN34, shown an unfavorable value of binding energies of $-11.04 \mathrm{Kcal} / \mathrm{mol}$ and $-9.91 \mathrm{Kcal} / \mathrm{mol}$ respectively, due to some steric hindrance phenomenon existing in the molecules.

We have studied many other groups substituted at different positions into ODN in addition to the above substitutions. We found moderate binding energy values of ODN15 (-11.85 Kcal/mol) and ODN25 (-11.50 Kcal/ $\mathrm{mol}$ ), formed when $-\mathrm{F}$ atom was included into the linker (C) and the side ring (B), respectively. Mono substituted cyanide group at C- 6 in ODN37 $(-11.41 \mathrm{Kcal} / \mathrm{mol})$ reported a much better binding score to compare to $-\mathrm{CN}$ group at C-7 in ODN14 ((-10.42 Kcal/mol). The molecules ODN26 and ODN11 containing single -NO2 group at C-13 and C-7 position respectively showed better binding affinity $(-11.60 \mathrm{Kcal} / \mathrm{mol}$ to $-11.37 \mathrm{Kcal} / \mathrm{mol})$. Similarly, the molecule ODN16 and ODN10 containing single $-\mathrm{CF}_{3}$ group at C-6 and C-7 position showed unsatisfactory docking scores $(-10.17 \mathrm{Kcal} / \mathrm{mol}$ to $-9.87 \mathrm{Kcal} / \mathrm{mol})$. On the contrary, when $-\mathrm{CF}_{3}$ replaced $-\mathrm{O}$ of ODN at C-10 position, a surprisingly very good result was obtained in ODN38 $(-11.88 \mathrm{Kcal} / \mathrm{mol})$, probably higher interaction of active moiety (B) with the binding site of the receptor. For the same reason, the ligand ODN36 recorded high docking value $(-11.89 \mathrm{Kcal} / \mathrm{mol})$, when the amide group has been used in place of $-\mathrm{CF}_{3}$ following the previous procedure.

Moreover, ODN39 has shown an interesting result. Here, substitution was done by highly polar $-\mathrm{CH}(\mathrm{OH})$ $\mathrm{COOH}$ and it was supposed to give rise to better docking with the binding site of Hsp90. But surprisingly, ODN39 with an adequate number of $\mathrm{H}$-bond interactions (four in number) displayed poor performance $(-9.88 \mathrm{Kcal} / \mathrm{mol})$ due to steric hindrance.

\section{Selection of Ligands}

The compounds ODN1, ODN5, ODN7, ODN22, ODN25, ODN30 and ODN37 displayed binding energies (-11.41 $\mathrm{Kcal} / \mathrm{mol}$ to $-12.60 \mathrm{Kcal} / \mathrm{mol}$ ) more compared to precursor ODN (-11.31 Kcal $/ \mathrm{mol})$ and bandgap energies $\Delta \mathrm{E}_{\mathrm{HL}}(3.46$ ev to $4.27 \mathrm{ev}$ ) also less than that of ODN (4.29 ev) All of them have qualified pharmacophore studies ( HIA $>70 \%$, $\mathrm{BBB}<0.2,90 \AA 2<\mathrm{TPSA}<140 \AA 2$, drug score $>0.4$ ) and in silico toxicity tests. Hence, these seven ligands could be considered as potent molecules, but before that, these compounds have to be synthesized in the wet lab followed by in vivo tests.

Marine natural product oroidin, a viable source of bioactive compounds, has a promising inhibitory effect on Heat shock protein (Hsp90), and that leads us to pursue this study. Here we have designed a library of thirty-nine derivatives by incorporation of various functional groups in the active pharmacophore. We have categorized the whole work into three systemic studies : (1) electronic, quantum mechanical, and thermodynamical behaviors of molecules using DFT at UB3LYP level of basis set $6-311++G(d, p),(2)$ molecular docking study of each compound in the active site of Hsp90 $\beta$, (3) drug-likeness and toxicity prediction and compared the outcomes with the standard drug ODN.

Analysis of all the data available from these studies revealed that seven analogs ODN1, ODN5, ODN7, ODN22, ODN25, ODN30, and ODN37 have better chemical activity and docking capabilities than the precursor molecule (ODN) one. Excellent drug-like behavior and low toxicity studies screened them as more potent inhibitors than the oroidin. These seven computationally designed derivatives may have the advantageous therapeutic strategies for cancer treatment and could be developed as novel effective anticancer drug candidates in the future.

\section{ACKNOWLEDGEMENTS}

The researchers leading to these results are thankful to HPC of the University of North Bengal for providing computational facility and to the department of chemistry for various aids.

\section{Human AND Animal Rights}

No Animals/Humans were used for studies that are the basis of this research.

\section{REFERENCES}

1. World Health Organization. Health Topics: Cancer. ehttps://www. who.int/news-room/fact-sheets/detail/cancr (Accessed July 06, 2020) 
2. Abd El-Hack ME, Abdelnour S, Alagawany M, Abdo M, Sakr MA, Khafaga AF, Mahgoub SA, Elnesr SS, Gebriel MG. Microalgae in modern cancer therapy: current knowledge. Biomed. Pharmacother. 2019;111:42-50.

3. Jhaveri K, Taldone, Modi S, Chiosis G. Advances in the clinical development of heat shock protein 90 (hsp90) inhibitors in cancers. Biochim. Biophys. Acta. 2012;1823(3):742-755.

4. Vabulas RM, Raychaudhuri S, Hartl HM, Hartl FU. Protein folding in the cytoplasm and the heat shock response. Cold Spring Harb Perspect. Biol. 2010;2(12):a004390.

5. Gupta SC, Sharma A, Mishra M, Mishra RK, Chowdhuri DK. Heat shock proteins in toxicology: how close and how far?. Life Sci. 2010; 86(11-12):377-384.

6. Lee HJ, Ock CY, Kim SJ, Hahm KB. Heat shock protein: hard worker or bad offender for gastric diseases. Int. J. Proteomics. 2010; 2010:259163.

7. Chen B, Zhong D, Monteiro A. Comparative genomics and evolution of the HSP90 family of genes across all kingdoms of organisms. BMC Genomics. 2006;7:156.

8. Chen B, Piel WH, Gui L, Bruford E, Monteiro A.The HSP90 family of genes in the human genome: insights into their divergence and evolution. Genomics. 2005;86(6):627-637.

9. Goetz MP, Toft DO, Ames MM, Erlichman C. The Hsp90 chaperone complex as a novel target for cancer therapy. Ann. Oncol. 2003;14 (8):1169-1176.

10. Taipale M, Jarosz DF, Lindquist S. Hsp90 at the hub of protein homeostasis: emerging mechanistic insights. Nat. Rev. Mol. Cell. Bio. 2010; 11(7):515-528.

11. Trepel J, Mollapour M, Giaccone G, Neckers L. Targeting the dynamic hsp90 complex in cancer. Nat. Rev. Cancer. 2010;10(8):537-549.

12. Lillsunde KE, Tomašič T, Kikelj D, Tammela P. Marine alkaloid oroidin analogues with antiviral potential: A novel class of synthetic compounds targeting the cellular chaperone Hsp90. Chem Biol Drug Des. 2017;90(6):1147-1154.

13. Sawai A, Chandarlapaty S, Greulich H, Gonen M, Ye Q, Arteaga CL, Sellers W, Rosen N, Solit DB. Inhibition of Hsp90 down-regulates mutant epidermal growth factor receptor (EGFR) expression and sensitizes EGFR mutant tumors to paclitaxel. Cancer Res. 2008;68 (2):589-596.

14. Mohsin SK, Weiss HL, Gutierrez MC, Chamness GC, Schiff R, Digiovanna MP, Wang CX, Hilsenbeck SG, Osborne CK, Allred DC, Elledge R, Chang JC. Neoadjuvant trastuzumab induces apoptosis in primary breast cancers. J. Clin. Oncol. 2005; 23(11):24602468.

15. Duerfeldt AS, Blagg BSJ. Hsp90 inhibition: elimination of shock and stress. Bioorg. Med. Chem. Lett. 2010;20(17):4983-4987.

16. Prodromou C, Nuttall JM, Millson SH, Roe SM, Sim TS, Tan D, Workman P, Pearl LH, Piper PW. Structural basis of the radicicol resistance displayed by afungal hsp90. Acs. Chem. Biol, 2009; 4(4):289-297.

17. Chiosis G, Lopes CE, Solit D. Heat shock protein-90 inhibitors: a chronicle from geldanamycin to today's agents. Curr. Opin. Investig. Drugs. 2006;7(6):534-541.

18. Lees-Miller SP, Anderson CW. The human double-stranded dnaactivated proteinkinase phosphorylates the 90 -kda heat-shock protein, hsp90 alpha at two nh2-terminal threonine residues. J. Biol. Chem. 1989;264:17275-17280.

19. Kurokawa M, Zhao C, Reya T, Kornbluth S. Inhibition of apoptosome formation bysuppression of hsp90beta phosphorylation in tyrosine kinase-induced leukemias. Mol. Cell. Biol. 2008; 28:5494-5506.

20. Rong B, Yang S, Molecular mechanism and targeted therapy of Hsp90 involved in lung cancer: New discoveries and developments (Review). Int. J. Oncol. 2018;52(2):321-336.

21. Pires ES, Khole VV. A block in the road to fertility: autoantibodies to heat-shock protein 90-beta in human ovarian autoimmunity. Fertil. Steril. 2009; 92 (4):1395-1409.

22. Donnelly A, Blagg BSJ. Novobiocin and additional inhibitors of the hsp90 c-terminal nucleotide-binding pocket. Curr. Med. Chem. 2008;15:2702-2717.
23. Mehbub MF, Lei J, Franco C, Zhang W. Marine sponge derived natural products between 2001 and 2010: trends and opportunities for discovery of bioactives. Mar. Drugs. 2014;12(8):4539-4577.

24. Molinski TF, Dalisay DS, Lievens SL, Saludes JP. Drug development from marine natural products. Nat. Rev. Drug. Discov. 2009; 8(1):69-85.

25. Rentas AL, Rosa R, Rodríguez AD, De Motta GE. Effect of alkaloid toxins from tropical marine sponges on membrane sodium currents. Toxicon. 1995;33(4):491-497.

26. Al-Mourabit A, Potier P. Sponge's molecular diversity through the ambivalent reactivity of 2-aminoimidazole: a universal chemical pathway to the oroidin-based pyrrole-imidazole alkaloids and their palau'amine congeners. Eur. J. Org. Chem. 2001;2:237-243.

27. Hoffmann H, Lindel T. Synthesis of the pyrrole-imidazole alkaloids. Synthesis. 2003;12:1753-1783.

28. Al-Mourabit A, Zancanella MA, Tilvi S, Romo D. Biosynthesis, asymmetric synthesis, and pharmacology, including cellular targets, of the pyrrole-2-aminoimidazole marine alkaloids. Nat. Prod. Rep. 2011;28(7):1229-1260.

29. Davis RA, Fechner GA, Sykes M, Garavelas A, Pass DM, Carroll AR, Addepalli R, Avery VM, Hooper JNA, Quinn RJ. Dibromophakellin: an alpha2B adrenoceptor agonist isolated from the Australian marine sponge, Acanthella costata. Bioorg. Med. Chem. 2009; 17:2497-2500.

30. Henry SH, Bosch FX, Bowers JC. Aflatoxin, hepatitis and worldwide liver cancer risks. Adv. Exp. Med. Biol. 2002;504:229-233.

31. Estey E, Döhner H. Acute myeloid leukaemia. Lancet. 2006; 368(9550):1894-1907.

32. Tomašić T, Nabergoj D, Vrbek S, Zidar N, Jakopin Ž, Žula A, Hodnik Ž, Jukič M, Anderluh M, Ilaš J, Dolenc MS, Peluso J, Ubeaud-Séquier G, Muller CD, Mašič LP, Kikelj D. Analogues of the marine alkaloids oroidin, clathrodin, and hymenidin induce apoptosis in human HepG2 and THP-1 cancer cells. Med. Chem. Commun. 2015; 6:105-110.

33. Dyson L, Wright AD, Young KA, Sakoff JA, McCluskey A. Synthesis and anticancer activity of focused compound libraries from the natural product lead, oroidin. Bioorg. Med. Chem. 2014; 22(5): 1690-1699.

34. Kadam RU, Roy N. Recent trends in Drug-Likeliness Prediction: A comprehensive review of In Silico methods. Indian J. Pharm. Sci. 2009; 14:609-615.

35. Shoichet BK, McGovern SL, Wei B, Irwin JJ. Lead discovery using molecular docking. Curr. Opin. Chem. Biol. 2002;6:439-446.

36. Molegro Molecular Viewer (MMV) 2.5.0. CLC Bio, Qiagen Inc, software available at http://www.clcbio.com/products/ clc-drugdiscovery-workbench. 2012.

37. ACD/Structure Elucidator version 2018.1. Advanced Chemistry Development Inc. Toronto, ON, Canada, www.acdlabs.com. 2019.

38. Dreizler RM, da J. Providencia Density Functional Methods in Physics. Plenum Press, New York, 1985.

39. Parr RG, Yang W. Density-Functional Theory of Atoms and Molecules. Oxford University Press, New York, 1989.

40. Frisch MJ, Trucks GW, Schlegel HB, Scuseria GE, Robb MA, Cheeseman JR, Scalmani G, Barone V, Petersson GA, Nakatsuji H, Li X, Caricato M, Marenich AV, Bloino J, Janesko BG, Gomperts R, Mennucci B, Hratchian HP, Ortiz JV, Izmaylov AF, Sonnenberg JL, Williams-Young D, Ding F, Lipparini F, Egidi F, Goings J, Peng B, Petrone A, Henderson T, Ranasinghe D, Zakrzewski VG, Gao J, Rega N, Zheng G, Liang W, Hada M, Ehara M, Toyota K, Fukuda R, Hasegawa J, Ishida M, Nakajima T, Honda Y, Kitao O, Nakai H, Vreven T, Throssell K, Montgomery JA, Jr Peralta JE, Ogliaro F, Bearpark MJ, Heyd JJ, Brothers EN, Kudin K.N, Staroverov VN, Keith TA, Kobayashi R, Normand J, Raghavachari K, Rendell AP, Burant JC, Iyengar SS, Tomasi J, Cossi M, Millam JM, Klene M, Adamo C, Cammi R, Ochterski JW, Martin RL, Morokuma K, Farkas O, Foresman JB, Fox DJ. Gaussian, Inc. Wallingford CT, Gaussian 16, Revision C.01. 2016.

41. Stephens P, Devlin F, Chabalowski C, Frisch MJ. Ab initio calculation of vibrational absorptionand circular dichroism spectra using density functional force fields. J. Phys. Chem. 1994; 98:1162311627. 
42. Lee C, Yang W, Parr RG. Development of the Colle-Salvetti correlation-energy formula into afunctional of the electron density. Phys. Rev. B Condens. Matter. 1988;37(2):785-789.

43. Morris GM, Huey R, Lindstrom W, Sanner MF, Belew RK, Goodsell DS, Olson AJ. Autodock4 and AutoDockTools4: automated docking with selective receptor flexiblity. J. Computational Chemistry. 2009; 16:2785-2791.

44.Sanner MF. Python: A Programming Language for Software Integration and Development. J. Mol. Graph Mod. 1999;17(1):57-61.

45. Rastogi SC, Mmendiratta N, Rastogi P. Bioinformatics Methods and Applications Genomics, proteomics and Drug Discovery: Structural Biology and Virtual screening for Drug Discovery. 4th ed, PHL Learning Private Limited, Delhi, 2015, pp. 426-444.

46. Fleming I. Frontier Orbital and Organic Chemical Reactions. John Wiley and Sons, New York, 1976.

47. Reed, J.L. Electronegativity: Chemical hardness I. J. Phys. Chem. A. 1997;101(40):7396-7400.

48. Barim E, Akman F. Synthesis. characterization and spectroscopic investigation of N-(2- acetylbenzofuran-3-yl) acrylamide monomer: Molecular structure, HOMO-LUMO study, TD-DFT and MEP analysis. Journal of Molecular Structure. 2019;1195:506-513.

49. Pauling L. The nature of the chemical bond and the structure of molecules and crystals: an introduction to modern structural chemistry. Cornell university press, 1960.
50. Parr RG, Yang W. Density functional approach to the frontierelectron theory of chemical reactivity. J. Am. Chem. Soc. 1984; 106:4049-4050.

51. Lipinski CA, Lombardo F, Dominy BW, Feeney PJ. Experimental and computational approaches to estimate solubility and permeability in drug discovery and development settings. Adv. Drug. Deliver Rev. 2001;23:4-25.

52. Husain A, Ahmad A, Khan SA, Asif M, Bhutani R, Al-Abbasi FA. Synthesis. molecular properties, toxicity and biological evaluation of some new substituted imidazolidine derivatives in search of potent anti-inflammatory agents. Saudi Pharm. J. 2016;24:104-114.

53. Tiwary BK, Pathak RK, Pradhan K, Nanda AK, Bothra AK, Chakraborty R. Evaluation of drug candidature of some quinazoline4- $(3 \mathrm{H})$-ones as inhibitor of human dihydrofolatereductase enzyme: molecular docking and in silico studies. Int. J. Pharm. Pharm. Sci. 2014;6:393-400.

54. Lee SK, Lee IH, Kim HJ, Chang GS, Chung JE, No KT. The PreADME Approach: web-based program for rapid prediction of physicochemical, drug absorption and drug-like properties, Euro QSAR 2002 designing drugs and crop protectants: processes, problems and solutions. Blackwell Publishing Maldenh, USA, 2003, pp. 418-420.

55. Ding F, Peng W. Biological activity of natural flavonoids as impacted by protein flexibility: An example of flavanones. Molecular Bio. Systems. 2015;11 (4):1119-1133.

HOW TO CITE THIS ARTICLE: Sarkar S, Das RK. Selection the Drug Efficacy of Oroidin Derivatives as Hsp90 Inhibitors by Computer Aided Drug Design Method. Int. J. Pharm. Sci. Drug Res. 2020;12(6):630-651. DOI: 10.25004/IJPSDR.2020.120607 